\title{
GALAXY NUMBER COUNTS FROM THE SLOAN DIGITAL SKY SURVEY COMMISSIONING DATA ${ }^{1}$
}

\author{
NaOki Yasuda, ${ }^{2}$ Masataka Fukugita,,${ }^{3,4}$ Vijay K. Narayanan, ${ }^{5}$ Robert H. Lupton, ${ }^{5}$ Iskra Strateva, ${ }^{5}$ \\ Michael A. Strauss, ${ }^{5}$ Željko Ivezić, ${ }^{5}$ Rita S. J. Kim, ${ }^{5}$ David W. HogG,${ }^{4}$ David H. Weinberg, ${ }^{6}$ \\ Kazuhiro Shimasaku, ${ }^{7}$ Jon Loveday, ${ }^{8}$ James AnNis, ${ }^{9}$ Neta A. BahCall, ${ }^{5}$ Michael Blanton, ${ }^{9}$ Jon Brinkmann, ${ }^{10}$ \\ Robert J. BrunNer, ${ }^{11}$ Andrew J. ConNolly, ${ }^{12}$ István Csabai, ${ }^{13}$ Mamoru Doi, ${ }^{7}$ Masaru Hamabe, ${ }^{7}$ \\ SHIN-ICHI ICHIKAWA ${ }^{2}$ TAKASHI ICHIKAWA, ${ }^{14}$ DAVID E. JOHNSTON, ${ }^{15}$ G. R. KNAPP, ${ }^{5}$ \\ Peter Z. Kunszt, ${ }^{13}$ D. Q. Lamb ${ }^{15}$ Timothy A. McKay, ${ }^{16}$ Jeffrey A. MunN, ${ }^{17}$ Robert C. Nichol,${ }^{18}$ \\ Sadanori OKamura, ${ }^{7}$ Donald P. SchneIDer,${ }^{19}$ GyUla P. Szokoly,${ }^{20}$ Michael S. Vogeley,${ }^{21}$ \\ Masaru Watanabe, ${ }^{22}$ and Donald G. YorK ${ }^{15}$ \\ Received 2001 January 18; accepted 2001 May 16
}

\begin{abstract}
We present bright galaxy number counts in five broad bands $\left(u^{\prime}, g^{\prime}, r^{\prime}, i^{\prime}, z^{\prime}\right)$ from imaging data taken during the commissioning phase of the Sloan Digital Sky Survey (SDSS). The counts are derived from two independent stripes of imaging scans along the celestial equator, one each toward the northern and the southern Galactic cap, covering about 230 and $210 \mathrm{deg}^{2}$, respectively. A careful study is made to verify the reliability of the photometric catalog. For galaxies brighter than $r^{*}=16$, the catalog produced by automated software is examined against eye inspection of all objects. Statistically meaningful results on the galaxy counts are obtained in the magnitude range $12 \leq r^{*} \leq 21$, using a sample of 900,000 galaxies. The counts from the two stripes differ by about $30 \%$ at magnitudes brighter than $r^{*}=15.5$, consistent with a local $2 \sigma$ fluctuation due to large-scale structure in the galaxy distribution. The shape of the number counts-magnitude relation brighter than $r^{*}=16$ is well characterized by $N \propto 10^{0.6 m}$, the relation expected for a homogeneous galaxy distribution in a "Euclidean" universe. In the magnitude range $16<r^{*}<21$, the galaxy counts from both stripes agree very well and follow the prediction of the no-evolution model, although the data do not exclude a small amount of evolution. We use empirically determined color transformations to derive the galaxy number counts in the $B$ and $I_{814}$ bands. We compute the luminosity density of the universe at zero redshift in the five SDSS bands and in the $B$ band. We find $\mathscr{L}_{B}=2.4 \pm 0.4 \times 10^{8} L_{\odot} h \mathrm{Mpc}^{-3}$, for a reasonably wide range of parameters of the Schechter luminosity function in the $B$ band.
\end{abstract}

Key words: cosmology: observations

\section{INTRODUCTION}

The number counts of galaxies as a function of magnitude is one of the classical cosmological tests. It has been repeatedly studied over many years by many authors and has yielded valuable insights about the properties of galaxies and their evolution with redshift (see Sandage 1988 and Koo \& Kron 1992 for reviews). However, despite much effort, the normalization of the counts-magnitude relation for bright galaxies remains uncertain by as much as $50 \%$. This leads to uncertainties in the normalization of the galaxy luminosity function at zero redshift at the same level and therefore to uncertainties in the interpretation of data on galaxy evolution with redshift. The slope of the galaxy counts at bright magnitudes is also a matter of debate;

\footnotetext{
${ }^{1}$ Based on observations obtained with the Sloan Digital Sky Survey. Information available at http://www.sdss.org.

${ }^{2}$ National Astronomical Observatory, Mitaka, Tokyo 181-8588, Japan.

${ }^{3}$ Institute for Cosmic-Ray Research, University of Tokyo, Kashiwa 277-8582, Japan.

${ }^{4}$ Institute for Advanced Study, Olden Lane, Princeton, NJ 08540.

${ }^{5}$ Princeton University Observatory, Princeton, NJ 08544.

${ }^{6}$ Department of Astronomy, Ohio State University, Columbus, OH 43210.

${ }^{7}$ Department of Astronomy and Research Center for the Early Universe, School of Science, University of Tokyo, Tokyo 113-0033, Japan.

${ }^{8}$ Sussex Astronomy Centre, University of Sussex, Falmer, Brighton BN1 9QJ, UK.

${ }^{9}$ Fermi National Accelerator Laboratory, P.O. Box 500, Batavia, IL 60510.

${ }^{10}$ Apache Point Observatory, 2001 Apache Point Road, P.O. Box 59, Sunspot, NM 88349-0059.

${ }_{11}$ Department of Astronomy, California Institute of Technology, Pasadena, CA 91125.

12 Department of Physics and Astronomy, University of Pittsburgh, 3941 O'Hara Street, Pittsburgh, PA 15260.

${ }^{13}$ Department of Physics and Astronomy, Johns Hopkins University, Baltimore, MD 21218.

${ }_{14}$ Astronomical Institute, Tohoku University, Sendai 980-8578, Japan.

${ }^{15}$ Department of Astronomy and Astrophysics, University of Chicago, 5640 South Ellis Avenue, Chicago, IL 60637.

16 Department of Physics, University of Michigan, 500 East University, Ann Arbor, MI 48109-1120.

${ }^{17}$ US Naval Observatory, Flagstaff Station, P.O. Box 1149, Flagstaff, AZ 86002.

${ }_{18}$ Department of Physics, Carnegie Mellon University, 5000 Forbes Avenue, Pittsburgh, PA 15213-3890.

19 Department of Astronomy and Astrophysics, 525 Davey Laboratory, Pennsylvania State University, University Park, PA 16802.

${ }^{20}$ Astrophysikalisches-Institut Potsdam, Germany.

${ }^{21}$ Department of Physics, Drexel University, 3141 Chestnut Street, Philadelphia, PA 19104.

22 Japan Science and Technology Corporation, Tokyo 102-0081, Japan.
} 
some authors have claimed that it is steeper than the slope predicted by the no-evolution model (Maddox et al. 1990, but see also Weir et al. 1995) and have argued either for a local underdensity in the galaxy distribution or for a rapid evolution of the galaxy population since $z \approx 0.2$.

Bright galaxies are rare on the sky, and their number density varies because of large-scale structure. Hence, we require imaging surveys extending over large solid angles to obtain reliable galaxy counts at the bright end. For this reason, most bright galaxy counts have been based on photographic plate material from Schmidt telescopes (Maddox et al. 1990; Bertin \& Dennefeld 1997), for which it is notoriously difficult to obtain accurate photometric calibration. Surveys with CCD imagers (Hall \& Mackay 1984; Tyson 1988; Lilly, Cowie, \& Gardner 1991; Metcalfe et al. 1991) have made dramatic progress in determining the galaxy counts at faint magnitudes, but the field of view covered by these surveys is usually small, making it difficult to accurately measure the number counts at bright magnitudes. Even the ambitious surveys of Gardner et al. (1996) and Postman et al. (1998) are limited to 8.5 and $14.7 \mathrm{deg}^{2}$, respectively, much smaller than the fields surveyed using photographic plates. What is clearly needed is an imaging survey of the sky, using modern CCD detectors, covering at least several hundred square degrees.

The Sloan Digital Sky Survey (SDSS; York et al. 2000) consists of an imaging survey in five photometric bands of $\pi$ steradians of the northern sky, as well as a follow-up spectroscopic survey of roughly $10^{6}$ galaxies and $10^{5}$ quasars, complete within precisely defined selection criteria. Images of the sky are obtained by SDSS at the rate of 20 square degrees an hour. Thus, useful data to determine the galaxy counts at bright magnitudes may be obtained in a few nights of imaging. In this paper, we present galaxy number counts in the five SDSS passbands $u^{\prime}, g^{\prime}, r^{\prime}, i^{\prime}$, and $z^{\prime}$, using imaging scans taken during the commissioning phase of the survey. We derive the galaxy counts in the magnitude range $12.5<r^{*}<21$, from two patches of the sky along the celestial equator, one each toward the northern and the southern Galactic cap, covering an area of about 230 and $210 \mathrm{deg}^{2}$, respectively. We emphasize that the number counts of galaxies constitute a crucial verification of the photometric catalog derived from the imaging survey, in particular on the uniformity of photometric measurements with magnitude.

The outline of this paper is as follows. In $\S 2$, we give a brief description of the observations and the data. We describe the photometry of galaxies and the associated errors in $\S 3$. In $\S 4$, we describe the construction of the galaxy catalog in the northern equatorial stripe, from the full photometric catalog. We present the galaxy number counts in the five SDSS bands in $\S 5$. In $\S 6$, we convert our number counts to the $B$ and $I_{814}$ bands using empirical color transformations and compare them with existing galaxy count results in the literature. We calculate the luminosity density of the universe in the SDSS bands and in the $B$ band in $\S 7$. As a consistency test, we recalculate the normalization of the luminosity functions for the SDSS passbands, which are obtained from the spectroscopic survey of the SDSS project and are published prior to this paper (Blanton et al. 2000). In $\S 8$, we derive the galaxy counts from the southern equatorial stripe. We present the color distributions of galaxies in $\S 9$. We present our conclusions in $\S 10$.

\section{IMAGING OBSERVATIONS AND DATA}

The SDSS survey is carried out using a wide-field $2.5 \mathrm{~m}$ telescope, a large-format imaging camera (Gunn et al. 1998), two fiber-fed double spectrographs, and an auxiliary $0.5 \mathrm{~m}$ telescope for photometric calibration. The sky is imaged in five passbands, $u^{\prime}, g^{\prime}, r^{\prime}, i^{\prime}, z^{\prime}$, covering the entire optical range from the atmospheric ultraviolet cutoff in the blue to the sensitivity limit of silicon CCDs in the red (Fukugita et al. 1996). The imaging camera contains 30 photometric CCDs, arranged in six columns of five rows, each row corresponding to a different filter. The data are taken in timedelay-and-integrate (TDI, or drift-scan) mode at the sidereal rate along great circles on the sky, yielding a strip consisting of six very long and narrow scan lines, each 13.5 wide. The effective exposure time (i.e., the transit time of an object across a single chip) is $54.1 \mathrm{~s}$. The scan lines of a given strip do not overlap, but observing a second strip offset from the first strip by about 12 . 8 gives a filled stripe 2.5 wide. The scan line in five colors produced by each column of five CCDs is divided into $2048 \times 1489$ pixel frames with an overlap of 128 pixels between adjacent frames; a set of five frames covering a given region of sky (in the colors $u^{\prime}, g^{\prime}, r^{\prime}$, $i^{\prime}$, and $z^{\prime}$ ) is referred to as a field. The pixels are 0.396 square on a side, which satisfies the Nyquist limit for seeing greater than 1"0 full width at half maximum (FWHM; see Gunn et al. 1998 for technical details). The $0.5 \mathrm{~m}$ telescope observes standard stars to determine the atmospheric extinction on each night; the zero point of the $2.5 \mathrm{~m}$ telescope is determined by observing patches of sky that the $2.5 \mathrm{~m}$ is observing as well.

The scans of the northern equatorial stripe were obtained in two nights of SDSS commissioning observations on 1999 March 20 and 21, with the telescope parked on the celestial equator (SDSS runs 752 and 756). The total observation time was about $7 \mathrm{hr}$ in run 752 and about $8 \mathrm{hr}$ in run 756. The two runs together form a filled stripe. For $r^{\prime}<16$, we present the galaxy counts from data in the right ascension range over which a full stripe is completed by this pair of runs: $145^{\circ} .14<\alpha<236^{\circ} .42$ and $-1.26<\delta<1.26$ (all coordinates are in J2000.0), covering a total area of 230 $\operatorname{deg}^{2}$.

The imaging data used in this paper were taken before the commissioning of the current Photometric Telescope. Therefore, we calibrated the data by observing secondary patches in the survey area using a (now decommissioned) 61 $\mathrm{cm}$ telescope at the observatory site and by observing primary standard stars using the US Naval Observatory's 40 inch $(1 \mathrm{~m})$ telescope, with filter and CCD characteristics nominally identical to those at the SDSS Photometric Telescope. Since the transformation from the primary standard stars to the objects observed with the SDSS $2.5 \mathrm{~m}$ telescope has not yet been fully defined, we expect photometric errors of $\approx 0.05 \mathrm{mag}$ in each band with respect to the proper SDSS photometric system. Thus, in this paper, we will denote our magnitudes as $u^{*}, g^{*}, r^{*}, i^{*}$, and $z^{*}$ to emphasize the preliminary nature of our photometric calibration, rather than the notation $u^{\prime}, g^{\prime}, r^{\prime}, i^{\prime}$, and $z^{\prime}$ that will be used for the final SDSS photometric system. However, we will use the latter notation to refer to the SDSS photometric passbands themselves. Our magnitude system is based on the $A B_{95}$ system (Fukugita et al. 1996). The mean flux density over each broad passband is $\left\langle f_{v}\right\rangle=3631 \times 10^{-0.4 \mathrm{~m}} \mathrm{Jy}$.

Figure 1 shows the FWHM of the point-spread function 


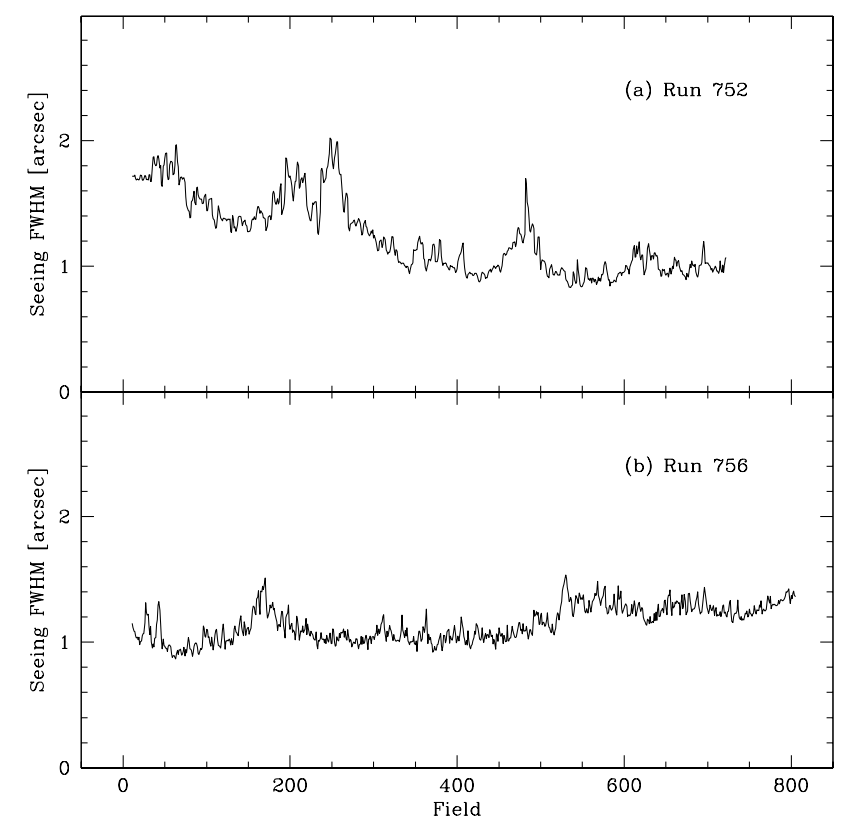

FIG. 1.-Field-to-field variation of seeing (FWHM) in the $r^{\prime}$ band in the third CCD column during TDI observations: (a) run 752, extending for 7 $\mathrm{hr}$; (b) run 756, extending for $8 \mathrm{hr}$.

(PSF) in the $r^{\prime}$ band (the seeing) in each field of the third CCD column. The PSF is fit to a pair of concentric circular Gaussians; the FWHM shown in this figure is that of this model. ${ }^{23}$ The top and bottom panels show the seeing variations in runs 752 and 756, respectively. The median PSF FWHM in the $r^{\prime}$ band was $1.4^{\prime \prime}$ in run 752 and 1..3 in run 756 , although the seeing varied substantially over the duration of the runs. The sky brightness also varied significantly during the runs. For example, in run 756 brightness of the $u^{\prime}$ and $g^{\prime}$ bands varied by $0.75 \mathrm{mag}$, that of $r^{\prime}$ by $0.45 \mathrm{mag}, i^{\prime}$ by $0.23 \mathrm{mag}$, and $z^{\prime}$ by $0.61 \mathrm{mag}$ during the scan for $8 \mathrm{hr}$. For the $z^{\prime}$ band, we recognize a $10 \%$ variation in a short timescale $(\approx 10$ minutes) in addition to a global change. The average sky brightness was $22.77,22.06,21.05,20.34$, and $19.18 \mathrm{mag} \operatorname{arcsec}^{-2}$ in $u^{\prime}, g^{\prime}, r^{\prime}, i^{\prime}$, and $z^{\prime}$, respectively.

\section{PHOTOMETRY OF GALAXIES}

The imaging data are processed with the PHOTOMETRIC PIPELINE (hereafter PHOTO; Lupton et al. 2001, see also York et al. 2000) ${ }^{24}$ specifically written for reducing the SDSS data. Each field is processed independently by PHOTO. Galaxies do not have sharp edges, nor unique profiles of surface brightness distributions, which makes it nontrivial to define a flux for each object. PHOTO measures a number of different types of magnitudes for each object. We present the galaxy counts using the Petrosian flux (Petrosian 1976), which is defined by

$$
F_{\mathrm{P}}=2 \pi \int_{0}^{k r_{\mathrm{P}}} I(r) r d r
$$

\footnotetext{
${ }^{23}$ Note that this FWHM measure gives a value somewhat smaller than that reported in some other SDSS papers, where the size of seeing is defined by the square root of the effective number of pixels calculated from the ratio of the first moment square to the second moment of the flux.

${ }^{24}$ The Sloan Digital Sky Survey Project Book is available at http:// astro.princeton.edu/PBOOK/welcome.htm.
}

where $I(r)$ is the surface brightness profile of the object in question, and $r_{\mathrm{P}}$ is the Petrosian radius satisfying

$$
\eta=\frac{I\left(r_{\mathrm{P}}\right)}{2 \pi \int_{0}^{r_{\mathrm{P}}} I(r) r d r /\left(\pi r_{\mathrm{P}}^{2}\right)} .
$$

In practice, this implicit equation for $r_{\mathrm{P}}$ is replaced with

$$
\eta=\frac{2 \pi \int_{0.8 \mathrm{r}_{\mathrm{P}}}^{1.25 r_{\mathrm{P}}} I(r) r d r /\left\{\pi\left[\left(1.25 r_{\mathrm{P}}\right)^{2}-\left(0.8 r_{\mathrm{P}}\right)^{2}\right]\right\}}{2 \pi \int_{0}^{r_{\mathrm{p}}} I(r) r d r /\left(\pi r_{\mathrm{P}}^{2}\right)},
$$

which yields robust measurements. Petrosian radius is independent of the distance of a galaxy and foreground reddening. Note that two parameters, $k$ and $\eta$, are needed to specify the Petrosian flux. We adopt $k=2$ and $\eta=0.2$ to optimize requirements. A small $\eta$ is preferred in order to minimize the discrepancy in apertures for galaxies with the exponential profile and those with the de Vaucouleurs profile. A small $\eta$ also makes the measurement of the Petrosian radius insensitive to seeing variations. On the other hand, consideration of signal-to-noise leads us to choose $\eta \geq 0.2$.

For every object, PHOTO calculates the Petrosian radius from aperture photometry using spline interpolation. With our adopted values of $k$ and $\eta$, the Petrosian flux is an integration over an aperture of radius of 3.6 half-light radii for an object with a de Vaucouleurs profile and 7.2 scale lengths for an object with an exponential profile. To quantify the difference between Petrosian and total magnitudes for different galaxy morphologies, we carry out simulations with an empirical model for the mix of galaxy morphology and the scale size (Shimasaku et al. 2001). The source of the other parameters used is explained in $\S 5$. We assume that the de Vaucouleurs profile is truncated at 5 half-light radii. ${ }^{25}$ We find that the Petrosian magnitude underestimates the total flux by $\approx 0.03 \pm 0.01$ mag (mean value) for this morphological mix, although the offset can be as large as 0.15-0.2 mag for face-on de Vaucouleurs profiles. The Petrosian radius is computed in all color bands, but we measure the Petrosian flux in any color band using the Petrosian radius for the $r^{\prime}$ band.

We have compared the photometry of objects that lie in the overlap regions between runs 752 and 756 . We find that the difference between the magnitudes of the same objects in these two runs taken under different seeing conditions is consistent with the errors in the magnitudes quoted by PHOTO. At $m=19$, the errors in Petrosian magnitudes are about $\pm(0.03-0.04) \mathrm{mag}$ in the $g^{\prime}, r^{\prime}, i^{\prime}$ bands and about 0.05 mag in the $u^{\prime}$ and $z^{\prime}$ bands.

We have studied the completeness limit of the SDSS imaging data, by comparison with simulated images and from comparison with deep Hubble Space Telescope (HST) images. Lupton et al. (2001) present a detailed study of the completeness limit of the SDSS imaging data. The comparison with deeper HST images shows that the SDSS imaging data are $50 \%$ complete to $r^{*}=22.5$, which agrees with the expectation from the simulation described above. This lim-

\footnotetext{
${ }^{25}$ With the untruncated de Vaucouleurs profile, $11 \%$ of the flux is distributed outside the aperture of this radius. The total flux of simulations is renormalized to the truncated de Vaucouleurs profile. Without the truncation the tail of the de Vaucouleurs profile causes a $2 \%-3 \%$ error in the local sky estimate.
} 
iting magnitude is substantially fainter than the $r^{*}=21$ limit to which we present the galaxy counts in this paper.

For the detection of objects PHOTO adopts a peakfinding algorithm using the matched-filter method, not only with the use of the PSF filter but also applied to $2 \times 2$ and $4 \times 4$ binned image. This algorithm is capable of detecting objects with quite low surface brightness. Our simulation shows that the $100 \%$ detection completeness is sustained at $r^{*}=21 \mathrm{mag}$ for galaxies with the scale length $5 \sigma$ larger than that of $M^{*}$ galaxies.

\section{CONSTRUCTION OF THE GALAXY CATALOG}

We have adopted two approaches to define a galaxy sample from the photometric catalog output by PHOTO. For bright magnitudes $\left(r^{*}<16\right)$, we have visually classified all objects in runs 752 and 756 into stars and galaxies. In $\S 4.3$, we compare the results of this visual classification with the results of the star-galaxy classification algorithm used in PHOTO. At fainter magnitudes $\left(16<r^{*}<21\right)$, we use the star-galaxy classification employed by PHOTO. In these faint magnitudes, PHOTO yields consistent classifications of objects in different colors.

\subsection{Selection of Objects from the Photometric Catalog}

Objects that lie in the overlap between adjacent scan lines in two strips of a stripe and those that lie in the overlap between adjacent frames appear more than once in the photometric catalog. Each of these detections is flagged as PRIMARY or SECONDARY, based on its position in the individual frames; the detections flagged as PRIMARY define a unique detection of each object. Therefore, we construct the galaxy catalog using only the PRIMARY objects.

PHOTO examines every detected object for multiple peaks; if they exist, the object is deblended (Lupton et al. 2001) into a hierarchy of component objects (children), and the parent is flagged as BLENDED. If a parent object has a large number of peaks, then only the 30 highest peaks are deblended. If the galaxy touches the edge of a frame, or if it is larger than half the size of a frame, it is not deblended any further, and the object is additionally flagged as NODEBLEND. ${ }^{26}$ Only isolated objects, child objects, and objects on which the deblender gave up are used in constructing the galaxy catalog.

\subsection{Star-Galaxy Classification}

PHOTO fits each object in all five colors to three model profiles convolved with the locally determined PSF, i.e., a point source, an exponential galaxy profile of arbitrary axial ratio, orientation and scale length, and a de Vaucouleurs galaxy profile of arbitrary axial ratio, orientation, and scale length. For each object, the best-fit model in the $r^{\prime}$ band is noted. The corresponding magnitude in the different bands, calculated using the parameters of the best-fit model in the $r^{\prime}$ band, is referred to as the model magnitude in that band. In principle, it is possible to use the likelihoods of these model fits to classify objects into stars and galaxies. We find empirically, however, that the star-galaxy classification is better using the following technique. Every object is classified as a star or a galaxy in each band, based on the difference between the model and PSF magnitudes. ${ }^{27}$ An

\footnotetext{
${ }^{26}$ Objects touching the edge of a frame will be deblended in the latest versions of PHOTO.
}

object is classified as a star in any band if the model magnitude and the PSF magnitude differ by no more than 0.145 mag, corresponding to the fluxes in the model fit and the PSF fit to the object satisfying the relation

$$
\frac{\text { Flux in PSF }}{\text { Flux in model }}<\frac{1}{0.875} \text {. }
$$

We have tested the accuracy of this star-galaxy classification algorithm by comparing our classifications of objects in SDSS images of the Groth strip against the classifications in the Medium Deep Survey catalog (MDS) constructed using WFPC2 parallel images from the HST (Ratnatunga et al. 1999 and references therein). The SDSS data were taken in 2000 May (runs 1468 and 1469). The FWHM of the median seeing was 1".4, slightly worse than the FWHM in runs 752 and 756 . We find that our stargalaxy classification correctly reproduces the MDS classifications for all objects (60 stars and 68 galaxies) with $r^{*}<20.5$. In the range $20.5<r^{*}<21,51$ out of 52 galaxies and 14 out of 14 stars are correctly classified. More details and tests of the star-galaxy classification algorithms used in PHOTO can be found in Lupton et al. (2001).

\subsection{Bright Galaxy Sample}

Although thestar-galaxy classification algorithm described in the previous subsection yields correct classifications in the range $16<r^{*}<21$ for the purpose of deriving reliable galaxy counts, there are reasons to suspect that this algorithm may run into problems at brighter magnitudes. For example, saturated pixels and diffraction spikes can compromise the model-fitting algorithm. ${ }^{28}$ In constructing the machine galaxy catalog from the photometric catalog, we reject all objects that contain saturated pixels (over the entire sky, only a handful of galaxies with active nuclei are expected to be saturated in the SDSS images). However, this rejection will also eliminate real galaxies that are blended with saturated stars. Hence, in order to calibrate the performance of the star-galaxy classification algorithm employed by PHOTO and to understand how well the deblender measures the magnitudes of galaxies with substantial internal substructure, we have visually examined the images of all objects brighter than $r^{*}=16$ in the stripe defined by runs 752 and 756, covering an area of about 230 $\mathrm{deg}^{2}$. At these bright magnitudes and large galaxy sizes, we expect our visual classification to be essentially perfect. Hence, we can use the visual galaxy catalog to quantify the completeness and the contamination fraction of the galaxy catalog constructed by PHOTO, which we refer to as the machine galaxy catalog. In this region, PHOTO classifies 27,304 objects as nonstellar (these include saturated stars) and 86,137 objects as stars.

Table 1 presents the results of comparing the visual and the machine galaxy catalogs at $r^{*}<16$. Column (1) lists the range of observed magnitudes (not yet corrected for reddening) in the $r^{\prime}$ band. Columns (2) and (3) show the

\footnotetext{
${ }^{27}$ PSF magnitude is computed for all objects by fitting a twodimensional PSF model, which is a continuously varying function of the position on the frame as determined from bright stars in the data.

${ }^{28}$ Obviously saturated pixels are rejected from fitting, but marginally saturated pixels may affect the model fitting. We are still working on subtracting the diffraction spikes from saturated stars in the current version of PHOTO.
} 
TABLE 1

Comparison of the Visual and Machine Galaxy Catalogs AT BRIGHT MAGNITUdeS

\begin{tabular}{ccccc}
\hline \hline $\begin{array}{c}\text { Magnitude } \\
\text { Range } \\
(1)\end{array}$ & $\begin{array}{c}\text { Visual } \\
\text { Counts } \\
(2)\end{array}$ & $\begin{array}{c}\text { Machine } \\
\text { Counts } \\
(3)\end{array}$ & $\begin{array}{c}\text { Visual } \\
\text { Only } \\
(4)\end{array}$ & $\begin{array}{c}\text { Machine } \\
\text { Only } \\
(5)\end{array}$ \\
\hline $9.5-10.0 \ldots \ldots$. & 1 & 0 & 1 & 0 \\
$10.0-10.5 \ldots \ldots$. & 0 & 0 & 0 & 0 \\
$10.5-11.0 \ldots \ldots$ & 1 & 1 & 0 & 0 \\
$11.0-11.5 \ldots \ldots$. & 4 & 1 & 3 & 0 \\
$11.5-12.0 \ldots \ldots$ & 4 & 5 & 0 & 1 \\
$12.0-12.5 \ldots \ldots$ & 4 & 3 & 1 & 0 \\
$12.5-13.0 \ldots \ldots$ & 10 & 14 & 0 & 4 \\
$13.0-13.5 \ldots \ldots$ & 30 & 32 & 3 & 5 \\
$13.5-14.0 \ldots \ldots$. & 58 & 75 & 4 & 21 \\
$14.0-14.5 \ldots \ldots$ & 101 & 114 & 12 & 25 \\
$14.5-15.0 \ldots \ldots$. & 257 & 279 & 15 & 37 \\
$15.0-15.5 \ldots \ldots$. & 517 & 548 & 24 & 55 \\
$15.5-16.0 \ldots \ldots$ & 987 & 1024 & 30 & 67 \\
Total: & 1974 & 2096 & 93 & 215 \\
\hline
\end{tabular}

number of objects in that magnitude bin that are classified as galaxies by visual inspection and by the machine galaxy catalog, respectively. Column (4) is the number of visually selected galaxies that are not included in the machine galaxy catalog. Column (5) shows the opposite case; the objects selected as galaxies in the machine galaxy catalog that are not real galaxies upon visual inspection.

There are 93 objects ( $5 \%$ of the sample) that are classified as galaxies by visual inspection but are not present in the machine galaxy catalog (the galaxies in column [4] of Table 1). These galaxies are not included in the machine galaxy catalog for the following reasons: (1) 48 galaxies are not selected because they are blended with saturated stars; (2) 45 galaxies are not classified in the $r^{\prime}$ band since they contain an interpolated pixel (i.e., a bad column or a cosmic ray) at or near the center, a condition that causes PHOTO to classify an object as UNKNOWN. ${ }^{29}$ Among these 45 galaxies, 43 are correctly classified as galaxies in both $g^{\prime}$ and $i^{\prime}$ bands. Hence, these galaxies would have been included in the machine galaxy catalog if we had selected as galaxies those objects that PHOTO classifies as galaxies in at least two of the three bands, $g^{\prime}, r^{\prime}$, and $i^{\prime}$.

Similarly, there are 215 objects that are classified as galaxies in the machine catalog, but are not confirmed to be real galaxies upon visual inspection (the objects in column [5] of Table 1). These objects are included in the machine galaxy catalog for the following reasons: (1) 108 objects are classified as galaxies in the $r^{\prime}$ band, but are classified as stars in all the other color bands. These objects are either single stars with slightly elongated shapes in the $r^{\prime}$ band or spurious objects caused by satellite trails. None of these objects are real galaxies. They would be excluded from the machine galaxy catalog if we required that the object be classified as a galaxy in at least two of the three bands $g^{\prime}, r^{\prime}$, and $i^{\prime}$; (2) 44 objects are double stars misclassified as galaxies; (3) 44 objects are redundant children arising from excessive deblending of other bright galaxies already present in the visual galaxy catalog; (5) three objects are groups of gal-

\footnotetext{
${ }^{29}$ Objects with interpolated pixels at the center are properly classified in later versions of PHOTO.
}

axies, whose individual members would be fainter than $r^{\prime}=16$ if they had been properly deblended; (4) four objects are selected as galaxies because of satellite trails and classified as galaxies in at least two of the three colors $g^{\prime}, r^{\prime}$, and $i^{\prime}$; (5) 12 objects are ghost images due to reflections of bright stars inside the camera.

We have reexamined the images of all of the 44 spurious children that resulted from excessive deblending, since such deblending problems will lead to underestimates of the flux of the parent galaxy. We have combined these 44 objects back to 31 parents and remeasured their Petrosian fluxes. We find that the error in magnitude resulting from this excessive deblending ranges from 0.1 to $1 \mathrm{mag}$, with a typical value of $0.5 \mathrm{mag}$. We use the corrected magnitudes for these 31 galaxies in the visual galaxy catalog. We note that the frequency of excessively deblending objects decreases at fainter magnitudes; in particular, there are no such objects in the $15<r^{*}<16$ bin, implying that excessive deblending is unlikely to be a problem fainter than 16 mag.

From Table 1, we see that the net statistical completeness of the machine galaxy catalog, defined as (galaxies common to both visual and machine catalogs)/(galaxies in visual catalog), is $95 \%$ for the $r^{*} \leq 16$ mag sample. However, if we require that all objects in the machine galaxy catalog be classified as galaxies in at least two out of the three bands, the completeness increases to $97 \%$. The major reason for missing real galaxies (incompleteness) in the machine galaxy catalog is that they are blended with saturated stars, while most of the spurious galaxy detections (contaminants) are either double stars or shredded pieces of brighter galaxies. The galaxy number counts obtained from the machine galaxy catalog matches the true galaxy counts to within $3 \%$ for the bright sample with $r^{*}<16$.

The comparison of the PHOTO outputs to the results of the visual inspection has uncovered a number of subtle problems with the photometry processing with PHOTO version 5.0.2 as of early 2000. Many of these problems are being fixed for a future version of the pipeline, which should have improved performance.

\subsection{Faint Galaxy Sample}

In the magnitude range $16<r^{*}<21$, we derive the galaxy counts using the machine galaxy catalog. At these magnitudes, we construct the machine galaxy catalog from the photometric catalog using the following criteria: (1) Only the PRIMARY detection of an object is included in the galaxy sample. (2) Based on the experience gained at bright magnitudes, we classify an object as a galaxy only if PHOTO classifies it as a galaxy in at least two of the three photometric bands, $g^{\prime}, r^{\prime}$, and $i^{\prime}$. (3) Objects that are blended with saturated stars are not included in the galaxy sample.

Figure 2 shows the distribution in the $g^{*}-r^{*}, r^{*}-i^{*}$ plane of all objects classified as stars and galaxies, in fields 251 to 300 of the third CCD column in run 756 (seeing FWHM is about $\left.1^{\prime \prime}\right) .{ }^{30}$ The colors are computed using the model magnitudes of objects in each band. The $g^{*}-r^{*}$ color is roughly constant for stars with $r^{*}-i^{*}>0.5$ (corresponding to stars cooler than $3500 \mathrm{~K}$ ) as the $g^{\prime}$ and $r^{\prime}$ bands are dominated by absorption from molecular bands,

\footnotetext{
${ }^{30} \mathrm{We}$ have not separated quasars from stars, so we expect about 50-100 quasars to be included in the star sample. In this paper, we have not studied the problem of blue compact galaxies.
} 

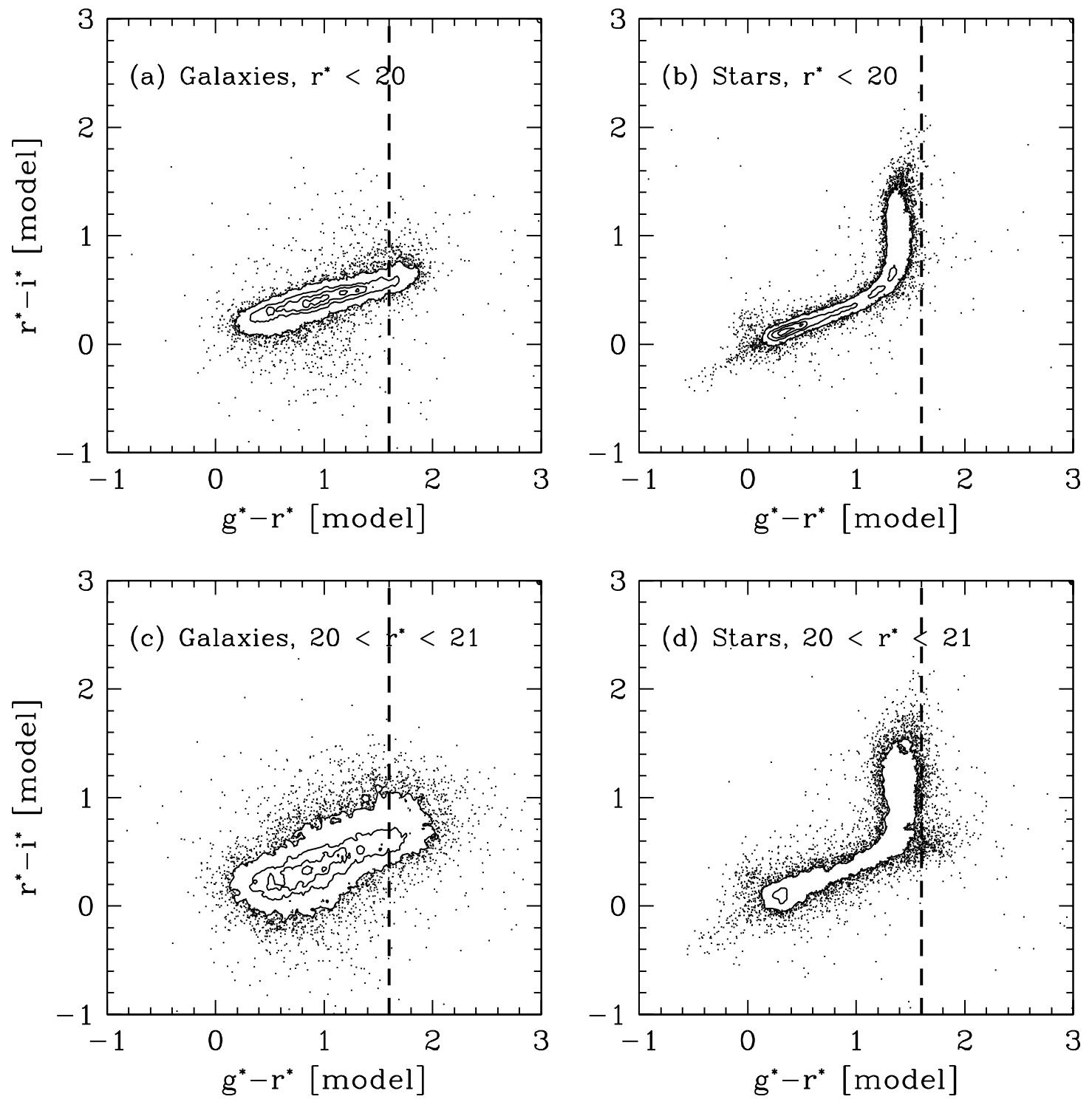

FIG. 2.-Distribution in the $g^{*}-r^{*}, r^{*}-i^{*}$ plane of all objects classified as galaxies and stars in fields 251 to 300 of the third CCD column of run 756 . (The star sample contains 50-100 quasars.) The inner parts of the distributions are shown as contours, linearly spaced in the density of objects in the color-color plane. $(a)$ and $(b)$ : Distribution of objects classified as galaxies and stars, respectively, in the magnitude range $16<r^{*}<20$. $(c)$ and $(d)$ : Corresponding objects in the magnitude range $20<r^{*}<21$. Objects in $(b)$ and $(d)$ with $g^{*}-r^{*}>1.6$ (dashed line) are real galaxies that are misclassified as stars, quasars, or stars with incorrect photometry.

especially from TiO. Hence, the objects with $g^{*}-r^{*}>1.6$ that are classified as stars (objects that lie to the right of the dashed line, Fig. $2 b$ and $2 d$ ) are quasars, stars with incorrect photometry, or real galaxies (including compact galaxies) that are misclassified as stars. Even if we assume that all objects with $g^{*}-r^{*}>1.6(1.7)$ are really galaxies, only $1.4 \%$ $(1 \%)$ of galaxies are being misclassified as stars in the magnitude range $16<r^{*}<20$ and $4 \%(2 \%)$ in the range $20<r^{*}<21$. Similarly, our tests of the star-galaxy classification algorithm using the images from the Groth strip (described in $\S 4.2$ ) show that at most a few percent of stars are being misclassified as galaxies at $r^{*}<21$. We have also compared the classifications of objects that lie in the overlap region between runs 752 and 756 . We found that the two classifications in these runs with different seeing were identical for $100 \%$ of objects at $r^{*}<20$, for $99 \%$ of objects in $20<r^{*}<20.5$, and for $97 \%$ of the objects in $20.5<r^{*}<21$. All these tests show that misclassification of objects does not pose a serious problem in deriving the galaxy counts at all magnitudes brighter than $r^{*}=21$.

\section{GALAXY NUMBER COUNTS IN THE SDSS BANDS}

Figure 3 shows the variation from one field to another of the number of stars and galaxies, averaged over all six CCD columns. The different panels show this variation in different bins in $r^{\prime}$ magnitude. Figure $3 a$ shows the objects in run 756 , while Figure $3 b$ shows those in run 752 . The abscissa in each panel is the field number. Note that a field corresponds to a $10^{\prime} \times 13^{\prime}$ region of sky, and the data shown are an average over six fields across the stripe. The global variation of the number of stars (left panels) reflects the change in Galactic latitude during the TDI scan over 7-8 $\mathrm{hr}$. The top left panel in each figure shows the variation of Galactic latitude over the duration of the scan. The number of gal- 

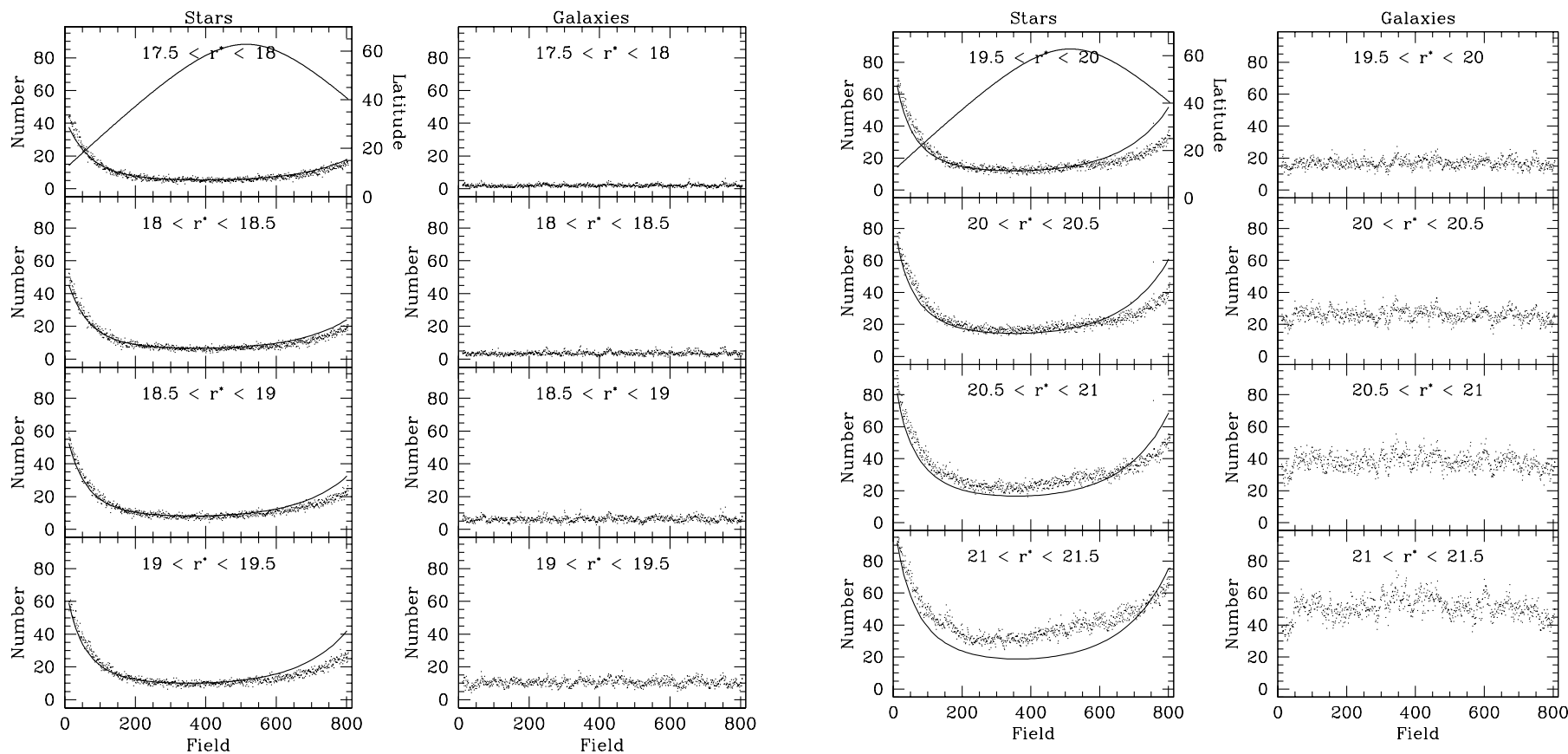

FIG. $3 a$
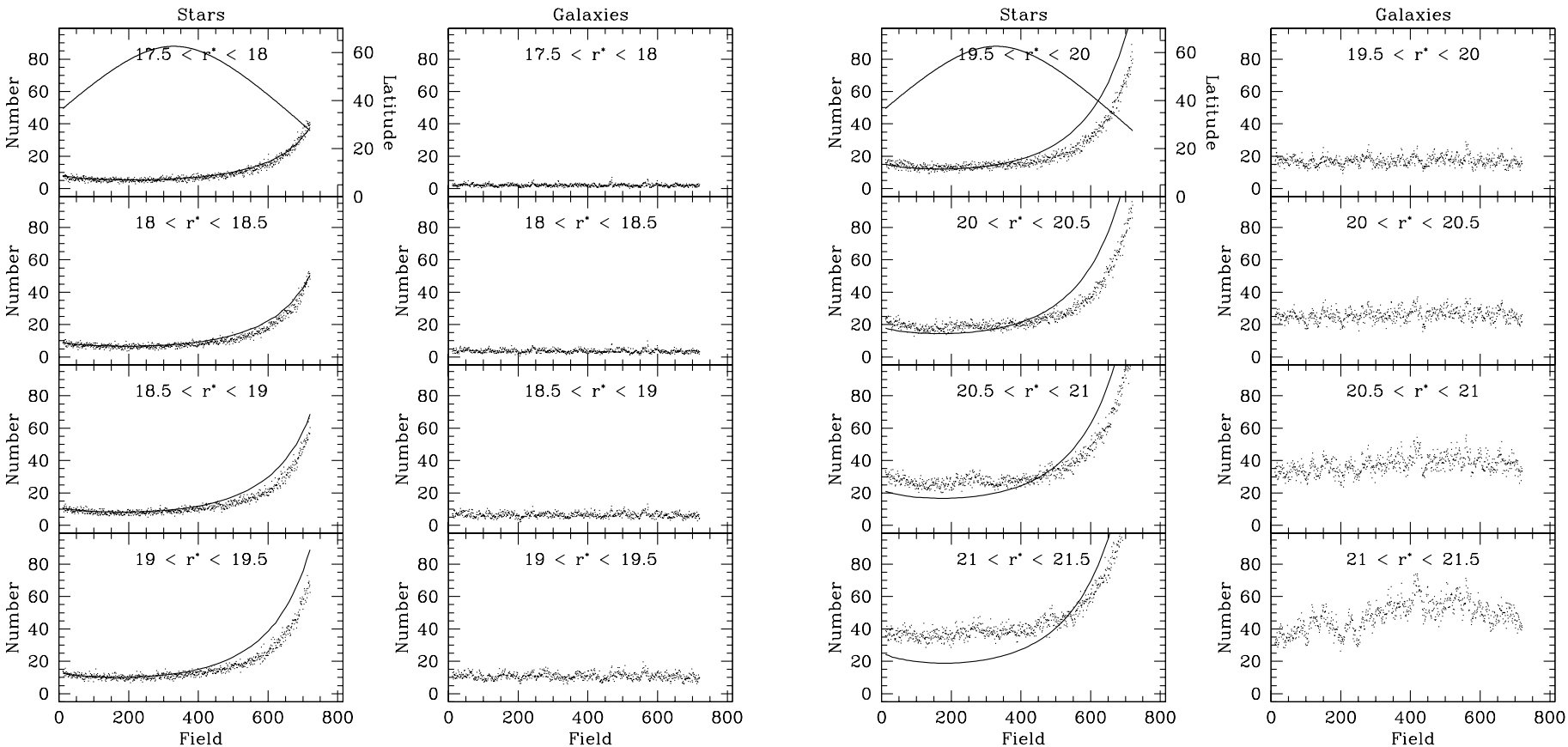

FIG. $3 b$

FIG. 3.-Field-to-field variation of the number of stars and galaxies in $(a)$ run 756 and $(b)$ run 752 , averaged over all six CCD columns. The solid curves in the left panels show the variation of star counts predicted by the Bahcall-Soneira model. The top left panel also shows the variation of Galactic latitude over the duration of this run.

axies varies from field to field because of both small-number statistics and small-scale clustering. However, there are no large-scale gradients in the galaxy distribution with field numbers in bins brighter than $r^{*}=21$, confirming that there is no serious contamination of stars to the galaxy counts in the magnitude range of interest. The galaxy counts in the faintest magnitude bin at $r^{*}>21$ could be systematically affected by galaxies being misclassified as stars. In particular, note the drop in the galaxy counts for $21<r^{*}<21.5$ in run 752 for small field numbers, where the seeing is poor, indicating that some fraction may not be detected and, more importantly, that some galaxies with low signal-to-noise ratio could be misclassified as stars. At the magnitude where we present the galaxy counts, there is no contamination from stars to galaxies, despite the fact that there is a higher ratio of stars to galaxies at brighter magnitudes. The solid curves in the left panels show the expected variation of star counts from the galaxy model of 
Bahcall \& Soneira (1980) using the color transformations derived from our work on primary standard stars (Smith et al. 2001)

$$
\begin{aligned}
& g^{*}=V+0.530(B-V)-0.075, \\
& r^{*}=V-0.438(B-V)+0.113 .
\end{aligned}
$$

We refer to Chen et al. (2001) and Finlator et al. (2000) for more detailed analysis of star counts. These empirical color transformations differ from the synthetic relations derived in Fukugita et al. (1996) by less than 0.03 mag in the constant terms. The model predictions and the observed star counts agree remarkably well at bright magnitudes $\left(r^{*} \leq 18.5\right)$ for all fields of the TDI scan. For $18.5<r^{*}<20.5$, the deviations of the observed star counts from the model predictions become increasingly apparent at large field numbers, which are pointing toward low Galactic latitudes in the direction of the Galactic center.

Figure 4 shows the galaxy number counts in 0.5 mag bins in the $r^{\prime}$ band in the northern equatorial stripe. We have co-added the counts in runs 752 and 756 for bins at $r^{*}<18$. At fainter magnitudes, we present the results from run 756 alone. We are certainly not limited by small-number statistics at $r^{*}>18 \mathrm{mag}$, and therefore we do not include the galaxy counts from run 752, where the seeing is worse. The filled points show the counts from the visual galaxy catalog, and the open points show the counts from the machine galaxy catalog. Star counts are also shown as crosses: the sharp drop seen at bright magnitudes $\left(r^{*}<14.5\right)$ is due to the fact that we cannot measure stars that saturate in the photometric CCD. Thus, our star counts are reliable in the

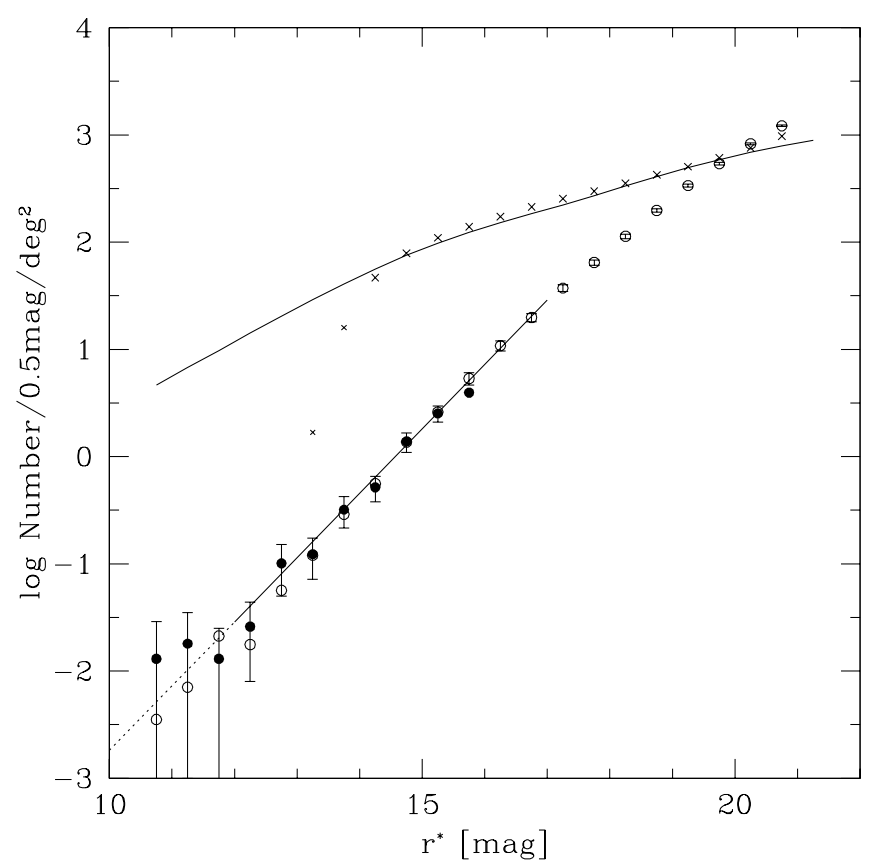

FIG. 4.-Number counts of galaxies as a function of magnitude in the $r^{\prime}$ band. Filled points show the galaxy counts from the visually inspected sample and open points from the machine-selected sample. The error bars include contributions from both shot-noise and large-scale structure (see text for details). The line segment shows the counts-magnitude relation expected for a homogeneous galaxy distribution in a universe with "Euclidean" geometry: $N\left(r^{*}\right)=A_{r * 1} 10^{0.6 r^{*}}$. The crosses show the observed star counts (small crosses at $r^{*}<14.5$ : data where stars saturate in the SDSS images and therefore suffer from incompleteness). The solid curve shows the prediction of the Bahcall-Soneira model.

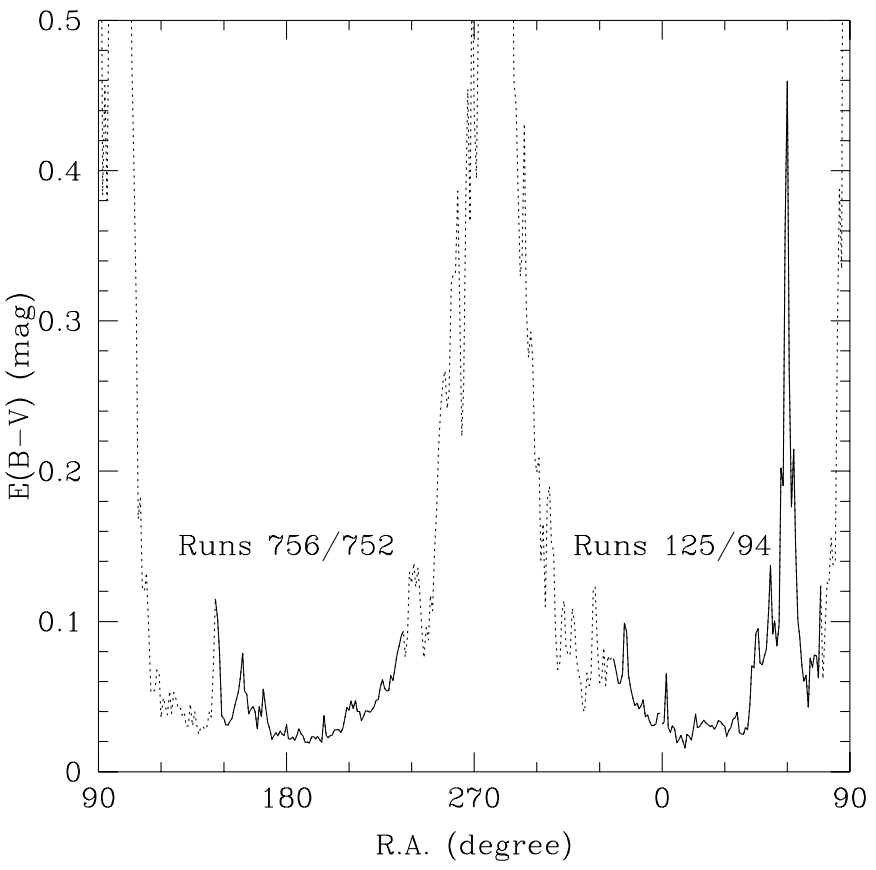

FIG. 5.-Selective extinction $E(B-V)$ from Schlegel et al. (1998) along the celestial equator. The solid curves show the ranges included in runs 752/756 (north) and runs 94/125 (south).

magnitude range $15<r^{*}<21$. We deredden the Petrosian magnitudes of galaxies using the Galactic extinction map of Schlegel et al. (1998), assuming a ratio of total to selective extinction in the $r^{\prime}$ band $R_{r}=2.75^{31}$ computed from a standard extinction law (Schlegel et al. 1998). For most of the sky region we have scanned, extinction is smaller than 0.1 mag [Fig. 5 shows selective extinction $E(B-V)$ along the celestial equator]. A smooth match between the galaxy counts from the visual and the machine galaxy catalogs is apparent; the two samples give almost identical galaxy counts in the range of magnitudes $14<r^{*}<15.5 .^{32}$ Hence, we derive the galaxy counts from the visual galaxy catalog at $r^{*} \leq 15.5$ and from the machine galaxy catalog at fainter magnitudes. Table 2 presents the galaxy counts in the five SDSS bands $u^{\prime}, g^{\prime}, r^{\prime}, i^{\prime}$, and $z^{\prime}$. We list the actual number of galaxies $\mathscr{N}$ detected in our sample in each $0.5 \mathrm{mag}$ bin (after dereddening the magnitudes of galaxies), together with the effective area in which we searched for galaxies in that bin. We note that the effective areas in the magnitude bins from the machine galaxy catalog are corrected for regions that are masked to avoid bright stars (amounting to about $0.4 \%$ of the total area).

The error bars on the galaxy counts data in Figure 4 include contributions from both Poisson noise and largescale structure in the galaxy distribution. The covariance between the galaxy counts in two magnitude bins is (Peebles 1993)

$$
<\delta N_{i} \delta N_{j}>=N_{i} \delta_{i j}^{K}+N_{i} N_{j} \iint \frac{w_{i j}\left(\theta_{i j}\right) d \omega_{i} d \omega_{j}}{\omega_{i} \omega_{j}},
$$

\footnotetext{
${ }^{31}$ Note that Petrosian magnitudes are easy to correct for extinction, unlike, for example, isophotal magnitudes (see the discussion in Santiago et al. 1996).

${ }^{32}$ The visually selected galaxy catalog is no longer complete in the magnitude bin $15.5<r^{*}<16$ after reddening corrections.
} 
TABLE 2

Number Counts of Galaxies IN the $u^{\prime}, g^{\prime}, r^{\prime}, i^{\prime}$, AND $z^{\prime}$ Color Bands For the Northern Equatorial StriPE ${ }^{a}$

\begin{tabular}{|c|c|c|c|c|c|c|c|c|c|c|c|}
\hline $\begin{array}{l}\text { Mag } \\
\text { Range } \\
(m \text { a g) }\end{array}$ & $\begin{array}{c}\text { Area } \\
\left(\operatorname{deg}^{2}\right)\end{array}$ & $\mathscr{N}_{u *}$ & $N_{u *}$ & $\mathscr{N}_{g *}$ & $N_{g *}$ & $\mathcal{N}_{r *}$ & $N_{r *}$ & $\mathscr{N}_{i *}$ & $N_{i *}$ & $\mathscr{N}_{z *}$ & $N_{z *}$ \\
\hline $11.0-11.5 \ldots \ldots$ & $228 \mathrm{v}$ & 1 & 0.004 & 3 & 0.013 & 4 & 0.018 & 3 & 0.013 & 2 & 0.009 \\
\hline $11.5-12.0 \ldots \ldots$ & $228 \mathrm{v}$ & 1 & 0.004 & 3 & 0.013 & 3 & 0.013 & 3 & 0.013 & 3 & 0.013 \\
\hline $12.0-12.5 \ldots \ldots$ & $228 v$ & 1 & 0.004 & 4 & 0.018 & 6 & 0.026 & 16 & 0.070 & 26 & 0.114 \\
\hline $12.5-13.0 \ldots \ldots$ & $228 \mathrm{v}$ & 3 & 0.013 & 4 & 0.018 & 23 & 0.101 & 28 & 0.123 & 33 & 0.144 \\
\hline $13.0-13.5 \ldots \ldots$ & $228 \mathrm{v}$ & 3 & 0.013 & 18 & 0.079 & 28 & 0.123 & 61 & 0.267 & 77 & 0.337 \\
\hline $13.5-14.0 \ldots \ldots$ & $228 \mathrm{v}$ & 5 & 0.022 & 28 & 0.123 & 73 & 0.320 & 97 & 0.425 & 145 & 0.635 \\
\hline $14.0-14.5 \ldots \ldots$ & $228 v$ & 11 & 0.048 & 59 & 0.258 & 118 & 0.517 & 244 & 1.07 & 352 & 1.54 \\
\hline $14.5-15.0 \ldots \ldots$ & $228 \mathrm{v}$ & 12 & 0.053 & 96 & 0.420 & 315 & 1.38 & 523 & 2.29 & 710 & 3.11 \\
\hline $15.0-15.5 \ldots \ldots$ & $228 v$ & 24 & 0.105 & 233 & 1.02 & 577 & 2.53 & 924 & 4.05 & $\ldots$ & $\ldots$ \\
\hline$\ldots$ & 283 & $\ldots$ & $\ldots$ & $\ldots$ & $\ldots$ & $\ldots$ & $\ldots$ & $\ldots$ & $\ldots$ & 1819 & 6.44 \\
\hline $15.5-16.0 \ldots \ldots$ & $228 \mathrm{v}$ & 53 & 0.232 & 452 & 1.98 & $\ldots$ & $\ldots$ & $\ldots$ & $\ldots$ & $\ldots$ & $\ldots$ \\
\hline$\ldots$ & 283 & $\ldots$ & $\ldots$ & $\ldots$ & $\ldots$ & 1513 & 5.35 & 2597 & 9.20 & 3734 & 13.2 \\
\hline $16.0-16.5 \ldots \ldots$ & 283 & 222 & 0.784 & 1170 & 4.14 & 3050 & 10.8 & 4806 & 17.0 & 7268 & 25.7 \\
\hline $16.5-17.0 \ldots \ldots$ & 283 & 555 & 1.96 & 2290 & 8.1 & 5607 & 19.8 & 9337 & 33.0 & 13601 & 48.1 \\
\hline $17.0-17.5 \ldots \ldots$ & 283 & 1393 & 4.92 & 4312 & 15.3 & 10532 & 37.3 & 16474 & 58.3 & 24426 & 86.4 \\
\hline $17.5-18.0 \ldots \ldots$ & 283 & 3121 & 11.0 & 7939 & 28.1 & 18239 & 64.5 & 29553 & 104.6 & 43837 & 155.1 \\
\hline $18.0-18.5 \ldots \ldots$ & 149 & 2823 & 18.9 & 6976 & 46.8 & 16890 & 113.2 & 27232 & 182.5 & 41673 & 279.4 \\
\hline $18.5-19.0 \ldots \ldots$ & 149 & 4898 & 32.9 & 11653 & 78.1 & 29443 & 197.4 & 47046 & 315.4 & 73714 & 494.1 \\
\hline $19.0-19.5 \ldots \ldots$ & 149 & 8105 & 54.4 & 19359 & 129.8 & 50206 & 336.5 & 77322 & 518.3 & 121858 & 816.9 \\
\hline $19.5-20.0 \ldots \ldots$ & 149 & 14799 & 99.3 & 31606 & 211.9 & 80324 & 538.4 & 121858 & 816.9 & 151616 & 1016 \\
\hline $20.0-20.5 \ldots \ldots$ & 149 & 28359 & 190.3 & 51000 & 341.8 & 123213 & 825.9 & 166314 & 1114 & 117358 & 786.7 \\
\hline $20.5-21.0 \ldots \ldots$ & 149 & 60618 & 406.8 & 82614 & 553.8 & 181428 & 1216 & 145706 & 976.7 & 64083 & 429.6 \\
\hline $21.0-21.5 \ldots \ldots$ & 149 & 111725 & 749.8 & 131641 & 882.4 & 242743 & 1627 & 65955 & 442.1 & 31661 & 212.2 \\
\hline
\end{tabular}

${ }^{\text {a }} \mathcal{N}$ is the number of galaxies in the sample, and $N$ is the normalized counts in units of counts per $0.5 \mathrm{mag}^{\mathrm{deg}}{ }^{-2}$. Results flagged with a " $\mathrm{v}$ " are based on the visual galaxy catalog $(\S 4)$, while the rest are based on the machine galaxy catalog. The galaxy counts at magnitudes fainter than 18 are based on run 756 alone.

where $N_{i}, N_{j}$ are the number of galaxies in bins $i$ and $j, \omega_{\mathrm{i}}$, $\omega_{\mathrm{j}}$ are the areas of the sky from which the galaxy numbers are derived, $\delta_{i j}^{K}$ is the Kronecker delta symbol, $w_{i j}(\theta)$ is the angular correlation function between the galaxies in bins $i$ and $j$, and the double integral extends over every pair of points in areas $\omega_{i}$ and $\omega_{j}$ that are separated by any angle $\theta_{i j}$. The first term is the contribution from Poisson noise and is included only in computing the variance in counts in a magnitude bin. The second term is the contribution from large-scale structure in the galaxy distribution. We use the covariance matrix of the galaxy counts in different magnitude bins in fitting for the normalization of the countsmagnitude relation.

In any two magnitude bins, we estimate the contribution from the second term as follows. We first assume that $w(\theta)$ can be represented as a power-law of the form $w(\theta)=A(m) \theta^{1-\gamma}$, where $\gamma=1.8$ is the slope of the two-point correlation function (Peebles 1993). We describe the details of computing the angular correlation function $w_{i j}(\theta)$ between galaxies in different magnitude bins in the Appendix. We perform the double integral

$$
I_{i j}=\iint \theta_{i j}^{1-\gamma} d \omega_{i} d \omega_{j}
$$

in a patch of sky $90^{\circ} \times 2.5$, roughly corresponding to the size and geometry of the stripe defined by runs 752 and 756, and use the same value of $I_{i j}$ for all possible pairs of geometries of the survey areas in different magnitude bins. We fix the amplitude of $w(\theta)$ assuming that $w(\theta=0.1)=0.1$ for galaxies in the magnitude range $18<r^{*}<18.5$, approximately the value measured from SDSS commissioning data itself (Scranton et al. 2000).
The line segment in Figure 4 shows a fit to the galaxy counts-magnitude relation expected in a homogeneous universe assuming Euclidean geometry for three-dimensional space (we refer to this as "Euclidean" geometry in the following text),

$$
N\left(m_{\lambda}\right)=A_{\lambda} 10^{0.6\left(m_{\lambda}-16\right)} .
$$

The observed galaxy counts are quite consistent with this line, with $A_{r *}=5.99 \pm 0.52(0.5 \mathrm{mag})^{-1} \mathrm{deg}^{-2}\left(\chi^{2}=13.9\right.$ for 9 degrees of freedom) for $12<r^{*}<17$ and even beyond this range. For $r^{*}<13$, the number of galaxies is quite small ( $N \leq 20$ in each bin), and there are large errors due to both Poisson noise and large-scale clustering.

In order to study the number counts in detail, we plot in Figure 6 the quantity $N\left(r^{*}\right) 10^{-0.6\left(r^{*}-16\right)}$, the observed galaxy number counts normalized by the shape of the counts-magnitude relation (hereafter called the growth rate) expected for a homogeneous galaxy distribution in a universe with "Euclidean" geometry. We also show the predictions of a no-evolution model, taking into account both cosmological corrections and $K$-corrections. The model is based on studies of the statistics of galaxy parameters in the $B$ band. We use a mix of E, S0, Sab, and Scd galaxies, in the ratio of morphological types given in Fukugita et al. (1998) and use the color shifts and $K$-corrections given in Fukugita et al. (1995). We assume that the luminosity function is characterized by a Schechter function with parameters $\alpha=-1.1$, and $M_{B}^{*}=-19.5+5 \log h$. The shape of the predicted curves depends only weakly on the model parameters. We show the predictions of the no-evolution model in three different cosmologies: $\left(\Omega_{m}, \Omega_{\Lambda}\right)=(1,0),(0.3,0)$, and $(0.3,0.7)$. We also show the predictions in a universe 


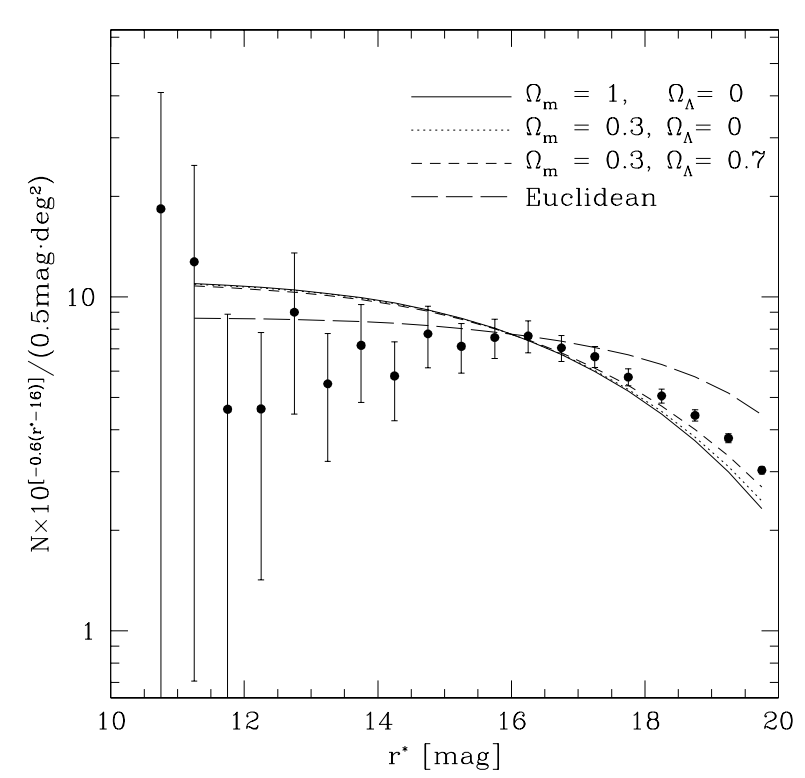

FIG. 6.-Galaxy counts-magnitude relation in the $r^{*}$ band, normalized by the expected growth rate in a "Euclidean" universe, i.e., $N\left(r^{*}\right)$ $\times 10^{\left[-0.6\left(r^{*}-16\right)\right]}$. Points show the observed galaxy counts, while the curves show the predictions of a no-evolution model in three different cosmologies. All the model curves are normalized to the amplitude $\tilde{A}_{r^{*}}$ derived using equation (10).

assuming "Euclidean" geometry for three space, but including $K$-corrections. Model curves are normalized to the amplitude $\widetilde{A}_{r^{*}}$ derived in equation (10).

The observed galaxy counts are consistent with the predictions of the no-evolution model for $r^{*}>16$, although the data permit some evolution. At $r^{*}<16$, the data mostly lie below the predictions of the no-evolution model, but they are mostly within the fluctuations expected from Poisson noise and large-scale structure in the galaxy distribution. We will show in $\S 6$ that this deficit of galaxies at bright magnitudes is much smaller in amplitude and much more local in volume than the local underdensity claimed by the APM group (Maddox et al. 1990). This small underdensity toward the northern Galactic cap is also not seen in our data toward the southern Galactic cap (analyzed in $\S 8$ below). It is therefore likely to be ascribed to a local fluctuation due to large-scale structure in the galaxy distribution.

We repeat the same analysis of the galaxy counts in the $u^{\prime}$, $g^{\prime}, i^{\prime}$, and $z^{\prime}$ passbands. We assume the ratio of total to selective extinction to be $R_{u}^{\prime}=5.16, R_{g}^{\prime}=3.79, R_{i}^{\prime}=2.09$, and $R_{z}^{\prime}=1.48$, respectively, taken from a standard extinction law (Schlegel et al. 1998). The results are presented in Figure 7 and Table 2. The error bars include contributions from both Poisson noise and large-scale structure. In each band we estimate $w(\theta)$ of galaxies in a magnitude bin from the corresponding value of $w(\theta)$ in the $r^{\prime}$ band, assuming a mean color for galaxies in that magnitude bin. The galaxy counts in the $g^{\prime}, i^{\prime}$, and $z^{\prime}$ passbands closely follow the $10^{0.6 m}$ law at bright magnitudes $(m<17-18)$, as we have seen with the counts in the $r^{*}$ band. The counts in the $u^{\prime}$ passband show some wiggle for $u^{*}<18 \mathrm{mag}$, and the local deficit is more conspicuous for brighter magnitudes. The reason is not clear to us.

As is evident from Figure 6, cosmological corrections and $K$-corrections have a nonnegligible effect on the galaxy counts at magnitudes even brighter than $r^{\prime}=16$ mag (as seen from the difference in shape between the curves corre-
TABLE 3

NORMALIZATION COEFFICIENTS OF THE $10^{0.6} \mathrm{~m}$ LAW, INCLUDING THE COSMOLOGICAL AND K CORRECTIONS

\begin{tabular}{ccc}
\hline \hline $\begin{array}{c}\text { Band } \\
(\lambda)\end{array}$ & $\begin{array}{c}\text { Fitting Range } \\
(\mathrm{mag})\end{array}$ & $\begin{array}{c}\tilde{A}_{\lambda} \\
{\left[(0.5 \mathrm{mag})^{-1} \mathrm{deg}^{-2}\right]}\end{array}$ \\
\hline$u^{*} \ldots \ldots$ & $12.0-19.5$ & $1.52 \pm 0.18$ \\
$g^{*} \ldots \ldots$ & $12.0-19.0$ & $4.95 \pm 0.29$ \\
$r^{*} \ldots \ldots$ & $12.0-18.0$ & $11.30 \pm 0.75$ \\
$i^{*} \ldots \ldots$ & $11.5-18.0$ & $18.66 \pm 0.90$ \\
$z^{*} \ldots \ldots$ & $11.5-18.0$ & $30.17 \pm 2.40$ \\
$B \ldots \ldots$ & $12.0-19.5$ & $2.98 \pm 0.17$ \\
\hline
\end{tabular}

sponding to different cosmologies and the "Euclidean" geometry), although in the data this effect is masked by fluctuations arising from Poisson noise and large-scale structure. To obtain the cosmologically meaningful normalization of the galaxy counts, we must take account of the cosmological and $K$-corrections. The fit must cover a magnitude range faint enough that local large-scale structure effects are avoided, but not so faint that evolutionary effects become important. In Figure $8 c$, we display with filled points the galaxy count data in the $r^{\prime}$ band divided by the no-evolution model prediction (i.e., the "Euclidean" growth factor and the cosmological and $K$-corrections), so that data should fall on a constant line if they are not affected by large-scale structure and evolution. Here we assume $\left(\Omega_{m}, \Omega_{\Lambda}\right)=(0.3,0.7)$, although the results are insensitive to cosmology (the open points are the counts data from southern celestial equator stripe, which are discussed in $\S 8$ ). We fit the data (only those denoted by filled points) taking account of Poisson noise and large-scale structure using the fitting range chosen to cover the plateau. We have carried out both full correlated and uncorrelated fits. The result of full correlated fit, however, turns out to give a rather poor fit to the number count data. ${ }^{33}$ Hence, we adopt the result from the uncorrelated fit. For example, for the $r^{\prime}$ band, we obtain the coefficient of the "Euclidean" growth rate in the bright limit, which we denote by $\tilde{A}_{r *}$,

$$
\tilde{A}_{r *}=11.30 \pm 0.75(0.5 \mathrm{mag})^{-1} \mathrm{deg}^{-2},
$$

where the error includes Poisson noise, large-scale structure, and the variation induced by the change of the faint end of the fitting range by \pm 1 mag. Figure 8 presents the analysis repeated for all passbands (including $B$ band, which we discuss in the next section). The $1 \sigma$ error band is indicated with dotted lines in the figure. We obtain the coefficient of the "Euclidean" growth rate in the bright limit $\tilde{A}$ in each band, which is given in Table 3, together with the range of magnitudes over which it was fit. We could not find a plateau for the $z^{\prime}$ band (to a lesser extent for the $i^{\prime}$ band), and hence the real error for $\widetilde{A}\left(z^{*}\right)$ would be larger than is quoted in the table.

\footnotetext{
${ }^{33}$ This implies that the smooth no-evolution model used here is not quite suitable to describe the data (correlated fits are sensitive to the adequacy of the fitting function). The values of $\tilde{A}$ from the full correlated fit are systematically lower by $0.6-1.3 \sigma$ (except for $u^{\prime}$ ) than the values from the uncorrelated fit, which we have given in the figure and table. The fitting function for $u^{\prime}$ is apparently inadequate, and we find a larger discrepancy between the two fits. For completeness, the values of $\tilde{A}$ from full correlated fit for the $u^{*}, g^{*}, r^{*}, i^{*}, z^{*}$, and $B$ bands are $0.91 \pm 0.10,4.73 \pm 0.24$, $10.47 \pm 0.57,17.48 \pm 0.81,28.71 \pm 1.17$, and $2.78 \pm 0.19$, respectively.
} 

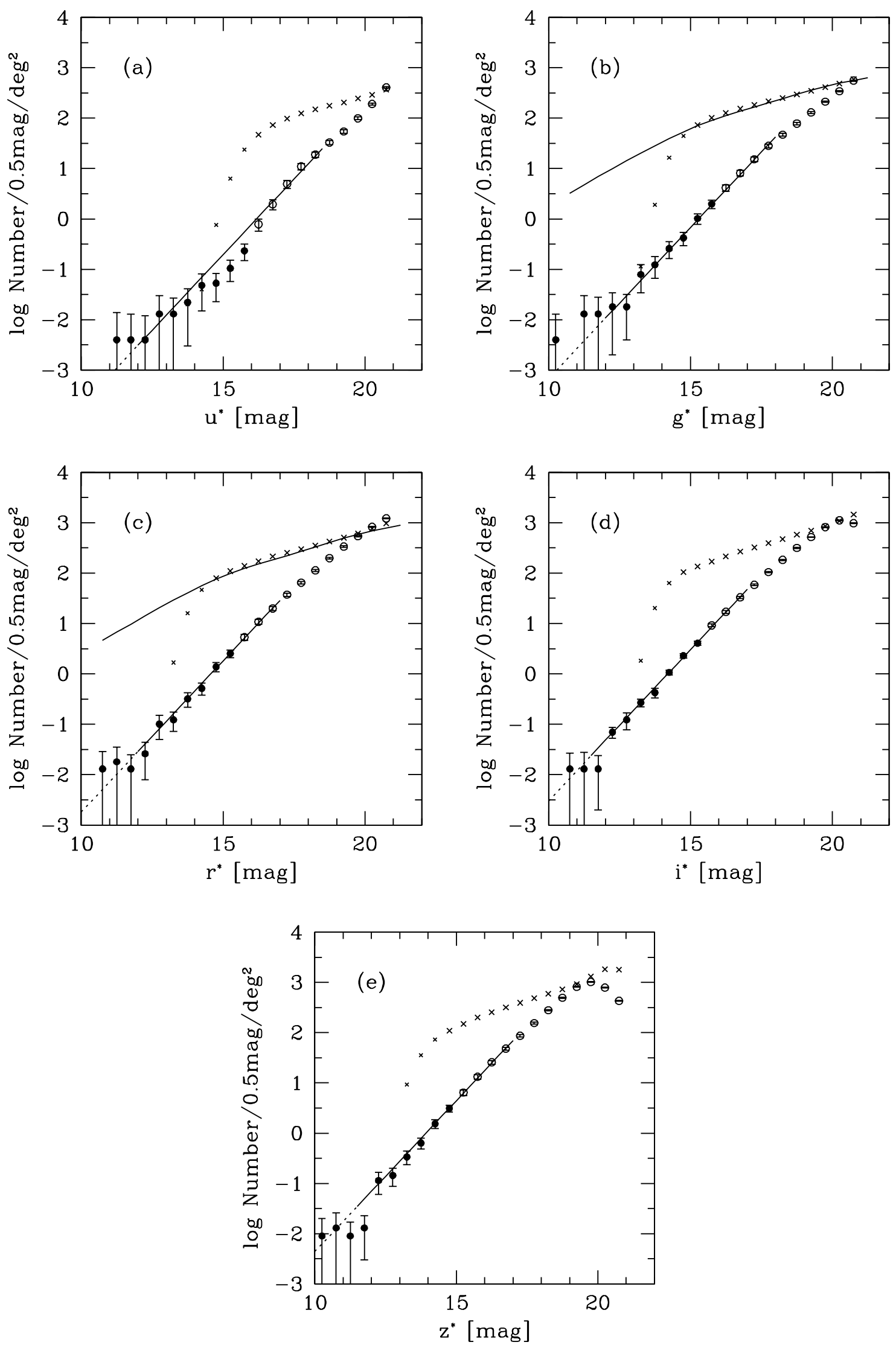

FIG. 7.-Number counts of galaxies as a function of magnitude in the five color bands $u^{\prime}, g^{\prime}, r^{\prime}, i^{\prime}$, and $z^{\prime}$ for the northern equatorial stripe. Filled points show the galaxy counts from the visually inspected sample, and open points from the machine-selected sample. The error bars include contributions from both shot-noise and large-scale structure (see text for details). The line segment shows the counts-magnitude relation expected in a homogeneous universe with "Euclidean" geometry: $N(m)=A_{1} 10^{0.6 m}$. The crosses show the observed star counts (small crosses: data where stars saturate in the image and therefore suffer from incompleteness), and the solid curve shows the prediction of the Bahcall-Soneira model. 

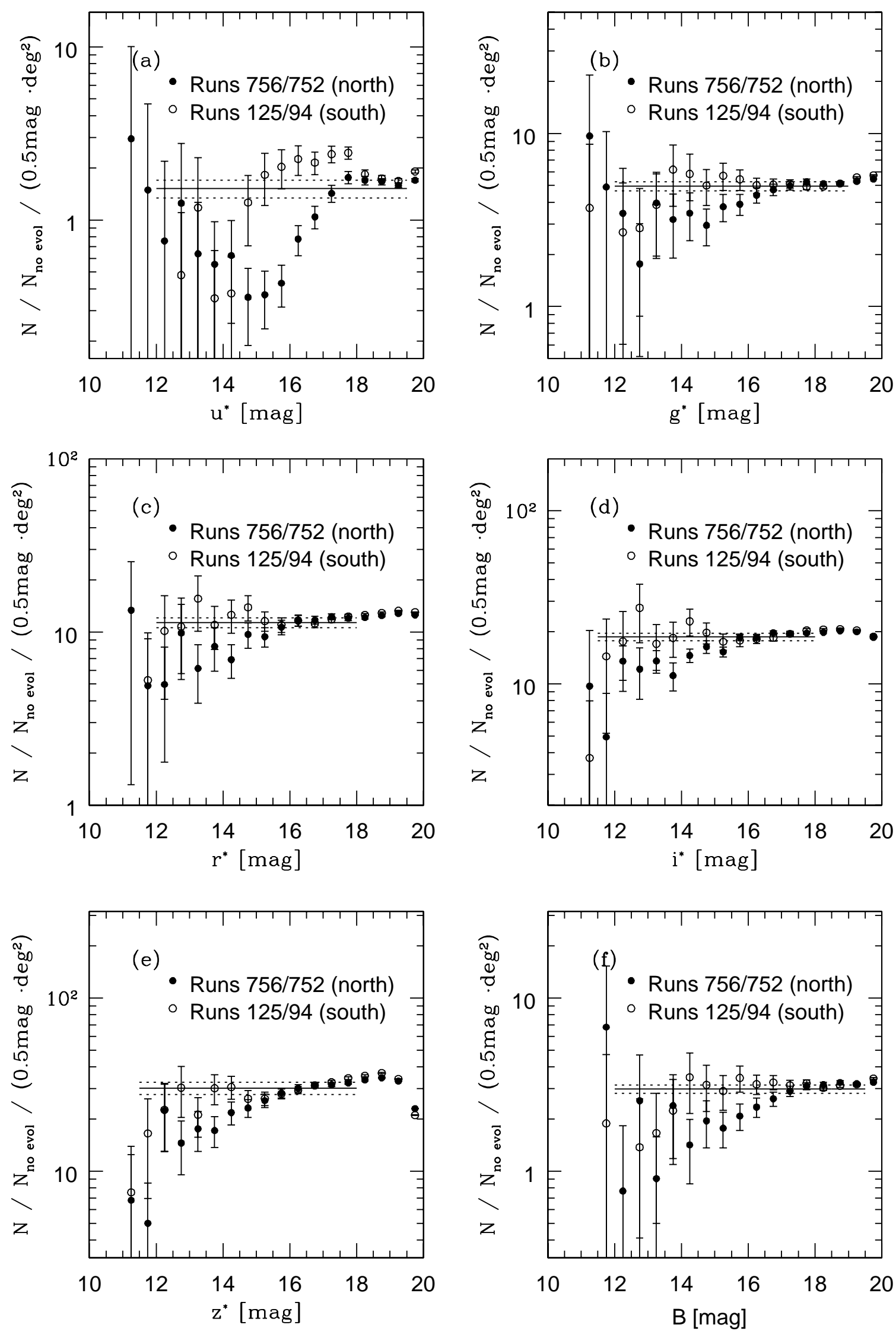

FIG. 8. - Galaxy counts-magnitude relation in the $u^{\prime}, g^{\prime}, r^{\prime}, i^{\prime}, z^{\prime}$, and $B$ bands, normalized by the no-evolution model prediction $N\left(\lambda^{\prime}\right)_{\text {no evol }}$. Filled points show the observed galaxy counts from the northern equatorial stripe, and open points are those from the southern equatorial stripe as discussed in $\S 8$. The solid lines correspond to $\tilde{A}_{\lambda *}$, and dotted lines show $1 \sigma$ error ranges. 
We do not discuss the detail of star counts in this paper. Extensive analyses of the star counts in the SDSS bands and their implications for models of Galactic structure are presented in Chen et al. (2001) and Finlator et al. (2000).

\section{GALAXY NUMBER COUNTS IN THE $B$ AND $I_{814}$ PASSBANDS}

The majority of results on galaxy number counts that exist in the literature have used the $B$ (or $B_{\mathrm{J}}$ ) band. Therefore, in this section, we convert our galaxy counts results to the Johnson-Morgan $B$-band photometric system and determine the normalization of the counts-magnitude relation. We adopt the color transformation

$$
B-g^{*}=0.482\left(g^{*}-r^{*}\right)+0.169,
$$

derived from preliminary standard star work (Smith et al. 2001) for the Landolt stars (Landolt 1992a, 1992b) with the standard deviation of $0.03 \mathrm{mag}$. We find that equation (11) provides a better match to the observed $B$-band photometry than an equivalent expression using the $u^{*}-g^{*}$ color. ${ }^{34}$ We have checked the accuracy of this transformation using colors synthesized from the spectra of galaxies. We use the spectroscopic energy distributions of galaxies from the spectrophotometric atlas of Kennicutt (1992) and carry out synthetic calculations of $u^{\prime}, g^{\prime}, r^{\prime}$, and $B$ magnitudes (see Fukugita et al. 1995 for more details). The $B$ magnitude calculated via equation (11) from synthetic $g^{\prime}$ and $r^{\prime}$ magnitudes agrees with the synthetic $B$ with an offset of -0.01 to $+0.04 \mathrm{mag}$ for elliptical and $\mathrm{Sb}$ galaxies at zero redshift (the plus sign means that the color transformation makes $B$ magnitude brighter than the synthetic value). The offset increases to $+0.1 \mathrm{mag}$ for late-type spirals. As we will see in $\S 9$ below, the mean color of our bright galaxy sample is $g^{*}-r^{*} \sim 0.7$, for which the offset nearly vanishes. The color transformation will in general also depend on the redshift of the galaxy, due to $K$-corrections. The accuracy degrades when the $4000 \AA$ spectral break moves deep into the $g^{\prime}$ band, but we find that the color transformation given by equation (11) works as well at $z \approx 0.2$ as at $z \approx 0$; for a galaxy at $z \approx 0.4$ the offset increases to about $0.2 \mathrm{mag}$. We apply the color transformation in equation (11) on a galaxy by galaxy basis and show the results only for $B<19.5$, as the fainter magnitude bins include a substantial fraction of galaxies with $z>0.3$.

Figure 9 shows the galaxy counts in the transformed $B$ band (after correcting for Galactic reddening), and Figure 10 shows the same quantity after normalizing by the "Euclidean" growth factor of $10^{0.6 m}$. The latter figure also shows the galaxy counts data in the $B$ band from the APM survey (Maddox et al. 1990) and from the surveys of Gardner et al. (1996) and Bertin \& Dennefeld (1997), together with the predictions of the no-evolution model. The photographic $b_{\mathrm{J}}$-band data of Maddox et al. (1990) and Bertin \& Dennefeld (1997) have been converted to $B$ band by Gardner et al. (1996). Note that each of these studies uses a different method to determine the magnitudes of galaxies, although there are attempts to make corrections to obtain

${ }^{34}$ One may consider the transformation to $B$ magnitude via $u^{*}$ and $g^{*}$, since the $B$ band is located between the $u^{*}$ and $g^{*}$ bands. However, this gives a larger offset except for late-type spiral galaxies; for example, for $g^{\prime}-r^{\prime} \simeq 0.7$ the offset is $0.06 \mathrm{mag}$. This offset results from the difference of $u^{\prime}$ band spectral features of stars and galaxies.

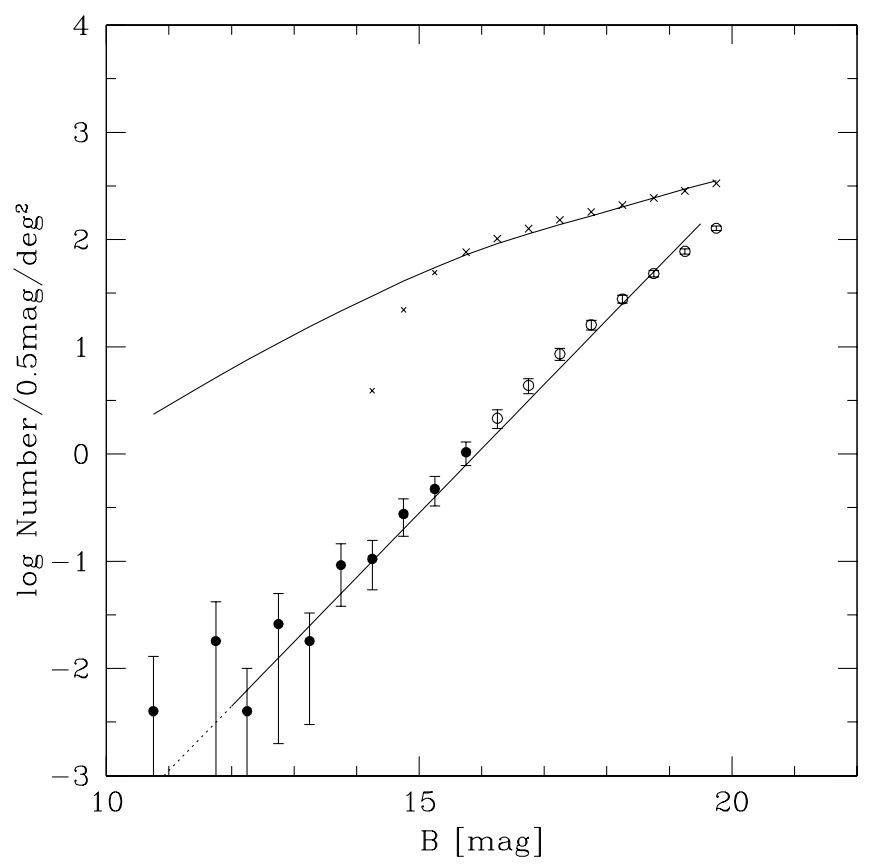

FIG. 9.-Number counts of galaxies as a function of magnitude in the $B$ band, using the color transformation given in eq. (11). Filled points show the galaxy counts from the visually inspected sample, and open points from the machine-selected sample. The error bars include contributions from both shot-noise and large-scale structure (see text for details). The line segment shows the counts-magnitude relation expected in a homogeneous universe with "Euclidean" geometry: $N(B)=A_{B} 10^{0.6 B}$. The crosses show the observed star counts (small crosses: data where stars saturate in the image and therefore suffer from incompleteness), and the solid curve shows the prediction of the Bahcall-Soneira model.

pseudo-total magnitudes; we refer the reader to the respective papers for details.

The shape of our galaxy counts fainter than $B=16.5$ agrees well with those of Gardner et al. (1996) and Bertin \&

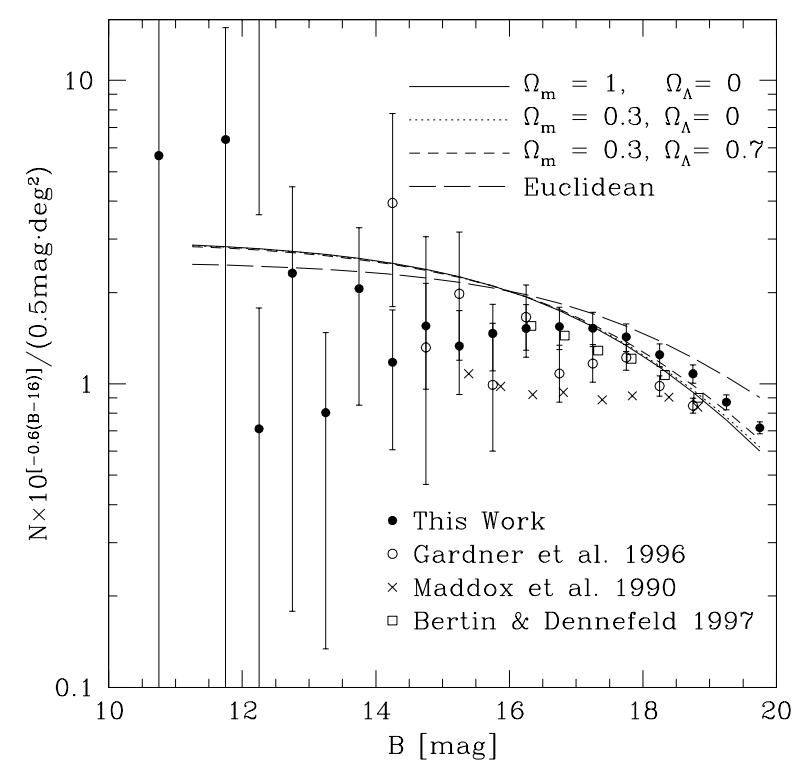

FIG. 10.-Galaxy counts-magnitude relation in the $B$ band normalized by the expected growth rate in a "Euclidean" universe, i.e., $N(B) \times 10^{[-0.6(B-16)]}$. Filled points show the observed galaxy counts from this work, while the curves show the predictions of a no-evolution model in three different cosmologies. All the model curves are normalized to the amplitude $\tilde{A}_{B}$ of the data. The open circles, crosses, and open squares show the data on galaxy counts in $B$ band from three previous studies. 


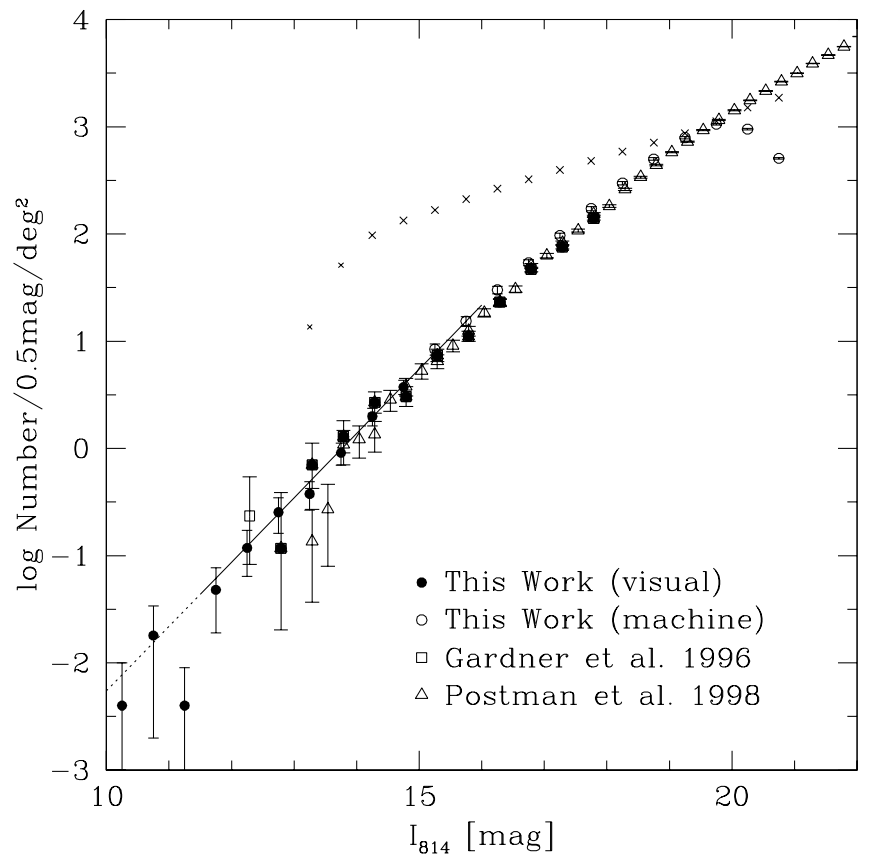

FIG. 11.-Number counts of galaxies as a function of magnitude in the $I_{814}$ band, using the color transformation given in eq. (12). Filled circles show the galaxy counts from the visually inspected sample, and open circles from the machine-selected sample. The error bars include contributions from both shot-noise and large-scale structure. The line segment shows the counts-magnitude relation expected in a homogeneous universe with "Euclidean" geometry: $N(I)=A_{I} 10^{0.61}$. The crosses show the observed star counts. The squares and triangles show galaxy counts from previous studies.

Dennefeld (1997), but our normalization is about $15 \%$ higher than theirs. On the other hand, the APM data lie substantially below our counts brighter than $18.5 \mathrm{mag}$. In particular, in the magnitude range $15<B<18$, our galaxy counts are on average a factor of 2 larger than the counts

TABLE 4

Number COUNTS OF GalaXIES IN THE $B$ AND $I_{814}$ BANDS IN THE NORTHERN EQUATORIAL STRIPE ${ }^{a}$

\begin{tabular}{|c|c|c|c|c|c|}
\hline $\begin{array}{l}\text { Mag. Range } \\
\text { (mag) }\end{array}$ & $\begin{array}{c}\text { Area } \\
\left(\operatorname{deg}^{2}\right)\end{array}$ & $\mathscr{N}_{B}$ & $N_{B}$ & $\mathscr{N}_{\mathbf{I}_{814}}$ & $N_{I_{814}}$ \\
\hline $11.0-11.5 \ldots \ldots$ & $228 v$ & 0 & 0.000 & 1 & 0.004 \\
\hline $11.5-12.0 \ldots \ldots$ & $228 \mathrm{v}$ & 4 & 0.018 & 11 & 0.048 \\
\hline $12.0-12.5 \ldots \ldots$ & $228 v$ & 1 & 0.004 & 27 & 0.118 \\
\hline $12.5-13.0 \ldots \ldots$ & $228 v$ & 6 & 0.026 & 58 & 0.254 \\
\hline $13.0-13.5 \ldots \ldots$ & $228 v$ & 4 & 0.018 & 86 & 0.377 \\
\hline $13.5-14.0 \ldots \ldots$ & $228 v$ & 21 & 0.092 & 208 & 0.911 \\
\hline $14.0-14.5 \ldots \ldots$ & $228 \mathrm{v}$ & 24 & 0.105 & 455 & 2.00 \\
\hline $14.5-15.0 \ldots \ldots$ & $228 v$ & 63 & 0.276 & 854 & 3.74 \\
\hline \multirow[t]{2}{*}{$15.0-15.5 \ldots \ldots$} & $228 v$ & 108 & 0.473 & $\ldots$ & $\ldots$ \\
\hline & 283 & $\ldots$ & $\ldots$ & 2377 & 8.41 \\
\hline \multirow[t]{2}{*}{$15.5-16.0 \ldots \ldots$} & $228 v$ & 237 & 1.04 & $\ldots$ & \\
\hline & 283 & $\ldots$ & $\ldots$ & 4347 & 15.5 \\
\hline $16.0-16.5 \ldots \ldots$ & 283 & 608 & 2.15 & 8501 & 30.1 \\
\hline $16.5-17.0 \ldots \ldots$ & 283 & 1230 & 4.35 & 15236 & 53.9 \\
\hline $17.0-17.5 \ldots \ldots$ & 283 & 2423 & 8.57 & 27316 & 96.7 \\
\hline $17.5-18.0 \ldots \ldots$ & 283 & 4526 & 16.0 & 48717 & 172.4 \\
\hline $18.0-18.5 \ldots \ldots$ & 149 & 4167 & 27.9 & 44432 & 297.8 \\
\hline $18.5-19.0 \ldots \ldots$ & 149 & 7192 & 48.2 & 74565 & 499.8 \\
\hline $19.0-19.5 \ldots \ldots$ & 149 & 11569 & 77.5 & 118000 & 791.0 \\
\hline $19.5-20.0 \ldots \ldots$ & 149 & 19017 & 127.4 & 157416 & 1055 \\
\hline
\end{tabular}

a See Table 2 for explanations. inferred from the APM survey. The shape of our countsmagnitude relation is also consistent with the predictions of the no-evolution model in the magnitude range $17<B<19$, while some deficit is seen in magnitudes brighter than $B=16$ arising from local large-scale structure in the galaxy distribution. Since the depth corresponding to the deficit is only $150 \mathrm{~h}^{-1} \mathrm{Mpc}^{-1}$ and our survey is only in a small patch on the sky, it appears that the deficit brighter than $B=16$ is consistent with the amplitude of large-scale structure fluctuations. We need not invoke an overall local underdensity of galaxies over a large solid angle. This is corroborated by the analysis for the southern stripe, as we discuss later. We note that the APM data remain flat over the range from 15.5 to $19 \mathrm{mag}$, while cosmological effects and $K$-correction should make the counts decrease significantly over this magnitude range; Maddox et al. (1990) interpret this as due to evolution. Our data do not show evidence for such a rapid "evolution" of the galaxy population to $z=0.2$ (approximately the redshift of an $L^{*}$ galaxy at $B=19$ ).

The star counts plotted in Figure 9 agree well with the predictions of the Bahcall-Soneira model in the range $15<B<19$. The agreement is a qualitative confirmation that our color transformation in equation (11) works well for stars.

We have also calculated the galaxy number counts in the $I_{814}$ passband (F814W) - the band in which much work has been done with the HST. From our standard star work (Smith et al. 2001), we find for the transformation to the Cousins $I_{\mathrm{C}}$ band that

$$
I_{\mathrm{C}}-i^{*}=-0.205\left(r^{*}-i^{*}\right)-0.382,
$$

with the standard deviation of $0.02 \mathrm{mag}$. We further apply color transformation from $I_{\mathrm{C}}$ to $I_{814}: I_{814}=I_{\mathrm{C}}+0.04$ according to Fukugita et al. (1995). This constant depends little on galaxy colors.

Figure 11 presents our galaxy counts in the $I_{814}$ band for $I_{814}<20$ mag. We also plot the data from Gardner et al. (1996) and Postman et al. (1998). The three independent data sets show good agreement up to an offset of about 0.05 mag, which might be ascribed to systematic errors in the different bands or to different definitions of the magnitudes of objects. Our numerical data for the galaxy counts are given in Table 4 for both $B$ and $I_{814}$ bands.

\section{LOCAL LUMINOSITY DENSITY OF THE UNIVERSE}

The normalization of the number counts $A$ of equation (9) is related to the parameters of the Schechter luminosity function (Schechter 1976) as

$$
A=0.7046 \frac{\omega}{3}\left(d_{16}^{*}\right)^{3} \Gamma(\alpha+5 / 2) \phi^{*},
$$

where $d^{*}=10 \mathrm{pc} \times 10^{0.2\left(16-M^{*}\right)}$, and $\omega$ is the solid-angle coverage of the sample. When we take account of cosmological effects and $K$-corrections, $A$ must be replaced with the coefficient $\tilde{A}$ obtained after corrections as we saw in $\S 5$. We then compute $\phi^{*}$ using equation (13).

Let us calculate the luminosity density of the universe in the $B$ band, which has long suffered from significant uncertainties. Since we do not determine $M^{*}$ and $\alpha$ in the $B$ band from our own data, we use the measurements from the literature (Efstathiou et al. 1988; Loveday et al. 1992; Zucca 1997; Ratcliffe 1998; Folkes et al. 1999). These parameters 
TABLE 5

LUMINOSITY DENSITY OF THE UNIVERSE IN DIFFERENT BANDS

\begin{tabular}{lccccc}
\hline \hline & & & & $\phi_{\lambda}^{*}$ \\
Band & $M_{\lambda}^{*}$ & $\alpha_{\lambda}$ & $\left(10^{-2} h^{3} \mathrm{Mpc}^{-3}\right)$ & $M_{\odot}$ & $\left(10^{8} L_{\odot} h \mathrm{Mpc}^{-3}\right)$ \\
\hline$u^{*} \ldots \ldots$ & $-18.34 \pm 0.08$ & $-1.35 \pm 0.09$ & $5.67 \pm 1.05$ & 6.38 & $6.30 \pm 0.85$ \\
$g^{*} \ldots \ldots$ & $-20.04 \pm 0.04$ & $-1.26 \pm 0.05$ & $1.81 \pm 0.16$ & 5.06 & $2.53 \pm 0.20$ \\
$r^{*} \ldots \ldots$ & $-20.83 \pm 0.03$ & $-1.20 \pm 0.03$ & $1.40 \pm 0.11$ & 4.64 & $2.60 \pm 0.21$ \\
$i^{*} \ldots \ldots$ & $-21.26 \pm 0.04$ & $-1.25 \pm 0.04$ & $1.27 \pm 0.10$ & 4.53 & $3.31 \pm 0.23$ \\
$z^{*} \ldots \ldots$ & $-21.55 \pm 0.04$ & $-1.24 \pm 0.05$ & $1.38 \pm 0.14$ & 4.52 & $4.60 \pm 0.43$ \\
$B \ldots \ldots$ & $-19.60_{-0.10}^{+0.20}$ & $-1.1 \pm 0.1$ & $2.05_{-0.30}^{+0.67}$ & 5.46 & $2.41_{-0.31}^{+0.46}$ \\
\hline
\end{tabular}

lie in the range $-19.7 \leq M_{B}^{*} \leq-19.4$ and $-1.0 \geq$ $\alpha \geq-1.2,{ }^{35}$ where $B_{\mathrm{J}}=B+0.1$ is applied to convert into the $B$ band when necessary. From $\tilde{A}_{B}=2.98 \pm 0.17$ $(B=12-19.5)$, we obtain $\phi^{*}=2.05 \pm 0.12_{-0.28}^{+0.66}$. Here the first error stands for that of $\tilde{A}$ and the second arises from uncertainties in the Schechter parameters.

We then calculate the luminosity density of the universe in the $B$ band. Note that the fractional error in luminosity density $(\mathscr{L})$ is much smaller than that in $\phi^{*}$ because the luminosity density goes as $\mathscr{L} \sim \phi^{*} L^{*} \Gamma(\alpha+2)$, while the number counts go roughly as $N(m) \sim \phi^{*} L^{* 3 / 2} \Gamma(\alpha+5 / 2)$, so that errors in $L^{*}, \alpha$, and $\phi^{*}$ largely cancel. We find that the error in $\mathscr{L}$ produced by uncertainties in the Schechter parameters is reduced to $3 \%$. The error in $\mathscr{L}$ is dominated by the error in the global number counts. We obtain

$$
\mathscr{L}_{B}=(2.41 \pm 0.39) \times 10^{8} L_{\odot} h(\mathrm{Mpc})^{-3}
$$

as our best estimate. Here we have applied a 3\% upward shift for the expected offset between our Petrosian flux and the total flux (see the discussion in $\S 3$ ) and included a 5\% error from provisional photometric calibration and uncertainties in the color transformation equations.

We also calculate the luminosity density of the universe in the five SDSS passbands $u^{\prime}, g^{\prime}, r^{\prime}, i^{\prime}$, and $z^{\prime}$ in a similar manner, adopting the values of the Schechter luminosity function parameters derived from the SDSS commissioning data itself (Blanton et al. 2000). Table 5 presents our estimates of $\phi_{\lambda}^{*}$ and $\mathscr{L}_{\lambda}$ in each band (in units of the solar luminosity in the respective band). We adopt $M_{u}(\odot)=$ $6.38 M_{g^{\prime}}(\odot)=5.06, \quad M_{r}(\odot)=4.64, \quad M_{i}(\odot)=4.53$, and $M_{z \prime}(\odot)=4.52$, which roughly correspond to $(B-V)_{\odot}=$ 0.63 (Fukugita et al. 2001). Here we assume the covariance of the errors of the Schechter fit

$$
\Delta\left(M_{B} \cdot \alpha\right) / \sqrt{\left(\Delta M_{B}\right)^{2} \cdot(\Delta \alpha)^{2}} \approx 1 .
$$

The values of $\phi_{\lambda}^{*}$ and $\mathscr{L}_{\lambda}$ are consistent with those of Blanton et al. (2000) within our $1 \sigma$ error for $r^{\prime}, i^{\prime}$, and $z^{\prime}$. Our values are higher for $u^{\prime}$ and $g^{\prime}$, but within $2 \sigma$.

\section{GALAXY NUMBER COUNTS IN THE SOUTHERN EQUATORIAL STRIPE}

We have also analyzed the galaxy counts in an additional equatorial stripe toward the southern Galactic cap. These data were taken on 1999 September 19 and 25 (SDSS runs 94 and 125). The first run scanned the sky for $5.3 \mathrm{hr}$ from

${ }^{35}$ Folkes et al. (1999) give $\alpha$ significantly steeper than this range. Their Schechter fit, however, poorly represents the step-wise maximal likelihood result, and the faint-end slope is significantly flatter than their $\alpha$ indicates. $\alpha=336.7$ to 56.28 , with declination extending from -0.93 to +1.17 . The second run scanned for $5.7 \mathrm{hr}$ from $\alpha=$ 350.6 to 76.7 with declination varying from -1.14 to +0.96 ; the two runs together make a filled stripe. The median seeing FWHM in the $r^{\prime}$ band was 1".4 in run 94 and 1.7 in run 125 , but the telescope was not well collimated during these runs. The total area covered in this southern equatorial stripe is $210 \mathrm{deg}^{2}$. We do not construct the visual galaxy catalog in this region, but instead use the machine galaxy catalog itself over the entire range of magnitudes $11<r^{*}<21$. For $r^{*}>18$, we present the galaxy counts from run 94 alone, since it has data taken under better seeing. We present the galaxy counts in the $u^{\prime}, g^{\prime}, r^{\prime}, i^{\prime}$, and $z^{\prime}$ bands in Figure 13 and Table 6.

Figure 12 shows the galaxy number counts in $r^{\prime}$ band normalized by the "Euclidean" growth factor of $10^{0.6 m}$, in both the northern (filled points) and the southern (open points) equatorial stripes. The error bars on the galaxy counts in both stripes include contributions from both Poisson noise and large-scale structure. The number counts from these two essentially independent regions of the sky are identical for $r^{*} \geq 16$ mag. At brighter magnitudes, the counts in the southern stripe are systematically larger than

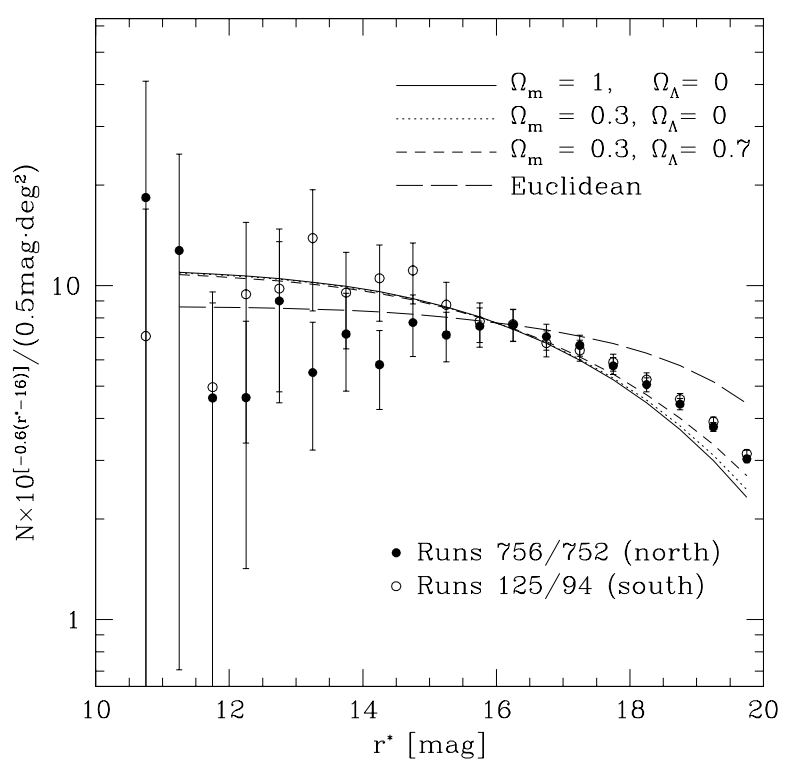

FIG. 12.-Galaxy counts-magnitude relation in the $r^{*}$ band normalized by the expected growth rate in a "Euclidean" universe, i.e., $N\left(r^{*}\right)$ $\times 10^{\left[-0.6\left(r^{*}-16\right)\right]}$. Filled points show the galaxy counts from the northern equatorial stripe, and open points show the counts from the southern equatorial stripe. The curves show the predictions of a no-evolution model in three different cosmologies. The model curves are normalized to the amplitude $\tilde{A}_{r *}$ of the data from the northern equatorial stripe. 
TABLE 6

Number Counts of GalaXies IN the $u^{\prime}, g^{\prime}, r^{\prime}, i^{\prime}$, AND $z^{\prime \prime}$ Bands IN the Southern Equatorial Stripe ${ }^{\mathrm{a}}$

\begin{tabular}{|c|c|c|c|c|c|c|c|c|c|c|c|}
\hline $\begin{array}{l}\text { Mag. } \\
\text { Range } \\
\text { (mag) }\end{array}$ & $\begin{array}{c}\text { Area } \\
\left(\mathrm{deg}^{2}\right)\end{array}$ & $\mathcal{N}_{u *}$ & $N_{u *}$ & $\mathscr{N}_{g *}$ & $N_{g *}$ & $\mathscr{N}_{r *}$ & $N_{r *}$ & $\mathscr{N}_{i *}$ & $N_{i *}$ & $\mathscr{N}_{z *}$ & $N_{z *}$ \\
\hline $14.0-14.5 \ldots \ldots$ & 209 & 6 & 0.029 & 91 & 0.436 & 196 & 0.939 & 352 & 1.686 & 453 & 2.17 \\
\hline $14.5-15.0 \ldots \ldots$ & 209 & 39 & 0.187 & 149 & 0.714 & 413 & 1.98 & 578 & 2.77 & 734 & 3.52 \\
\hline $15.0-15.5 \ldots \ldots$ & 209 & 108 & 0.517 & 322 & 1.54 & 650 & 3.11 & 968 & 4.64 & 1381 & 6.62 \\
\hline $15.5-16.0 \ldots \ldots$ & 209 & 228 & 1.09 & 576 & 2.76 & 1157 & 5.54 & 1842 & 8.83 & 2731 & 13.08 \\
\hline $16.0-16.5 \ldots \ldots$ & 209 & 475 & 2.27 & 984 & 4.71 & 2262 & 10.8 & 3477 & 16.65 & 5251 & 25.1 \\
\hline $16.5-17.0 \ldots \ldots$ & 209 & 843 & 4.03 & 1811 & 8.68 & 3962 & 18.9 & 6461 & 30.9 & 10063 & 48.2 \\
\hline $17.0-17.5 \ldots \ldots$ & 209 & 1733 & 8.29 & 3274 & 15.7 & 7525 & 36.0 & 12245 & 58.7 & 18648 & 89.3 \\
\hline $17.5-18.0 \ldots \ldots$ & 209 & 3197 & 15.3 & 5501 & 26.4 & 13823 & 66.2 & 22653 & 108.5 & 34586 & 165.7 \\
\hline $18.0-18.5 \ldots \ldots$ & 100 & 2056 & 20.6 & 4502 & 44.8 & 11724 & 116.8 & 18947 & 188.7 & 29486 & 293.7 \\
\hline $18.5-19.0 \ldots \ldots$ & 100 & 3426 & 34.3 & 7786 & 77.5 & 20435 & 203.5 & 32309 & 321.8 & 52662 & 524.5 \\
\hline $19.0-19.5 \ldots \ldots$ & 100 & 5752 & 57.5 & 13698 & 136.4 & 34895 & 347.5 & 52893 & 526.8 & 84069 & 837.3 \\
\hline $19.5-20.0 \ldots \ldots$ & 100 & 11178 & 111.8 & 22262 & 221.7 & 55831 & 556.0 & 81855 & 815.2 & 93168 & 927.9 \\
\hline
\end{tabular}

${ }^{\text {a }}$ See Table 2 for explanations.

those in the northern stripe. The southern stripe crosses the southern extension of the Pisces-Perseus supercluster; the enhanced counts are due to this well-known overdensity. The curves represent the predictions of the no-evolution model we described earlier.

The degree of disagreement in the galaxy counts at $r^{*}<16$ between the northern and the southern stripes indicates that the deficit of galaxies seen in the northern stripe is a local effect that depends on direction in the sky; i.e., the local universe to $100-200 \mathrm{Mpc}$ is clumpy. In the magnitude range $14<r^{*}<15.5$, there are $N_{1}=1010$ galaxies in the northern stripe, in an area of $\omega_{1}=230 \mathrm{deg}^{2}$. The rms fluctuation in this number due to large-scale structure is computed using equation (7) to be $\sigma=170$. In the same magnitude range, there are $N_{2}=1259$ galaxies in the southern stripe, in an area of $\omega_{2}=210 \mathrm{deg}^{2}$. The difference in counts between the two stripes is therefore $\mid N_{1}$ $-N_{2} \omega_{1} / \omega_{2} \mid=369$-plausibly a $2 \sigma$ fluctuation due to large-scale structure in the galaxy distribution. Thus, the difference in bright galaxy counts between the northern and southern equatorial stripes can be explained as arising from a large-scale structure fluctuation in the galaxy distribution. This supports our earlier observation in $\S 6$ that the deficit of galaxies at bright magnitudes in the northern stripe is due to large-scale structure fluctuations. We have to wait, however, for the end of the survey to draw definitive and quantitative conclusions on this point, as we quantify largescale structure over a much larger sample.

We refer to Figure 8 above for a comparison of the counts in the northern and southern equatorial stripes for the other color bands.

\section{COLOR DISTRIBUTIONS OF GALAXIES}

Figures 14 and 15 show the distribution of $g^{*}-r^{*}$ and $r^{*}-i^{*}$ colors of all galaxies in the third CCD column of run 756 as a representative sample. Dereddened Petrosian magnitudes are used to calculate colors, and color distributions are shown according to magnitude bin in $r^{*}$. Note that the Petrosian magnitude in each color band is computed using the Petrosian aperture calculated in the $r^{\prime}$ band. Therefore, the colors are measured through a consistent aperture in all bands. The mean $(\mu)$ and $\mathrm{rms}$ scatter $(\sigma)$ are indicated in each panel.

The mean of $g^{*}-r^{*}$ colors for the brightest bin is 0.71 , which is slightly $(0.04 \mathrm{mag})$ redder than expected from a spectroscopic synthesis calculation for a standard morphology mix (Fukugita et al. 1995). Figure 14 shows that the mean $g^{*}-r^{*}$ colors of galaxies become systematically bluer at bright magnitudes; the mean value of $g^{*}-r^{*}$ gradually shifts to 1.03 at $20<r^{*}<21$. The mean $r^{*}-i^{*}$ color of galaxies $(0.36$ in Fig. 15$)$ is consistent with what is expected from a synthesis calculation. This color stays roughly constant over the entire range of magnitudes $15<r^{*}<21$, as expected from the spectroscopic energy distributions of normal galaxies.

The dispersions in both the color distributions increase toward faint magnitudes. The scatter in color in bin $20<r^{*}<21$ is $0.52 \mathrm{mag}$ in $g^{*}-r^{*}$ and $0.35 \mathrm{mag}$ in $r^{*}-i^{*}$. These scatters are much larger than those expected from photometric errors alone; the photometric error in colors in this magnitude range is about $0.10 \mathrm{mag}$ in $g^{*}-r^{*}$ and 0.07 mag in $r^{*}-i^{*}$, both as quoted by PHOTO and from tests using simulated images. This broadening of the color distributions is expected from the $K$-corrections, which become increasingly different between galaxies of different morphological types with increasing redshift, and also from the broader range of redshifts that contribute to the colors in a given magnitude bin at fainter magnitudes.

\section{CONCLUSIONS}

We have presented the number counts of galaxies in five SDSS bands, $u^{\prime}, g^{\prime}, r^{\prime}, i^{\prime}$, and $z^{\prime}$, in the magnitude range $12<r^{*}<21$. We have first examined in detail the photometric catalog derived from the automated software PHOTO. We have carefully analyzed the bright galaxy sample by comparing the galaxy catalog produced by PHOTO against that produced by eye inspection. We have shown that the statistical completeness of the machinegenerated galaxy catalog is $97 \%$ for the sample with $r^{*}<16$ mag. However, $5 \%$ of objects in the machine galaxy catalog up to $r^{*}=16 \mathrm{mag}$ are not real galaxies. Toward the faint end, the imaging data are complete to $r^{*}=22.5 \mathrm{mag}$, and our star-galaxy classification is $100 \%$ accurate to $r^{*}=20$ mag. The misclassification rate is still a few percent at $r^{*}=21 \mathrm{mag}$. We expect that the performance will be improved in the future version of PHOTO.

We have then presented the number counts of galaxies in five SDSS bands $u^{\prime}, g^{\prime}, r^{\prime}, i^{\prime}$, and $z^{\prime}$ in the magnitude range 

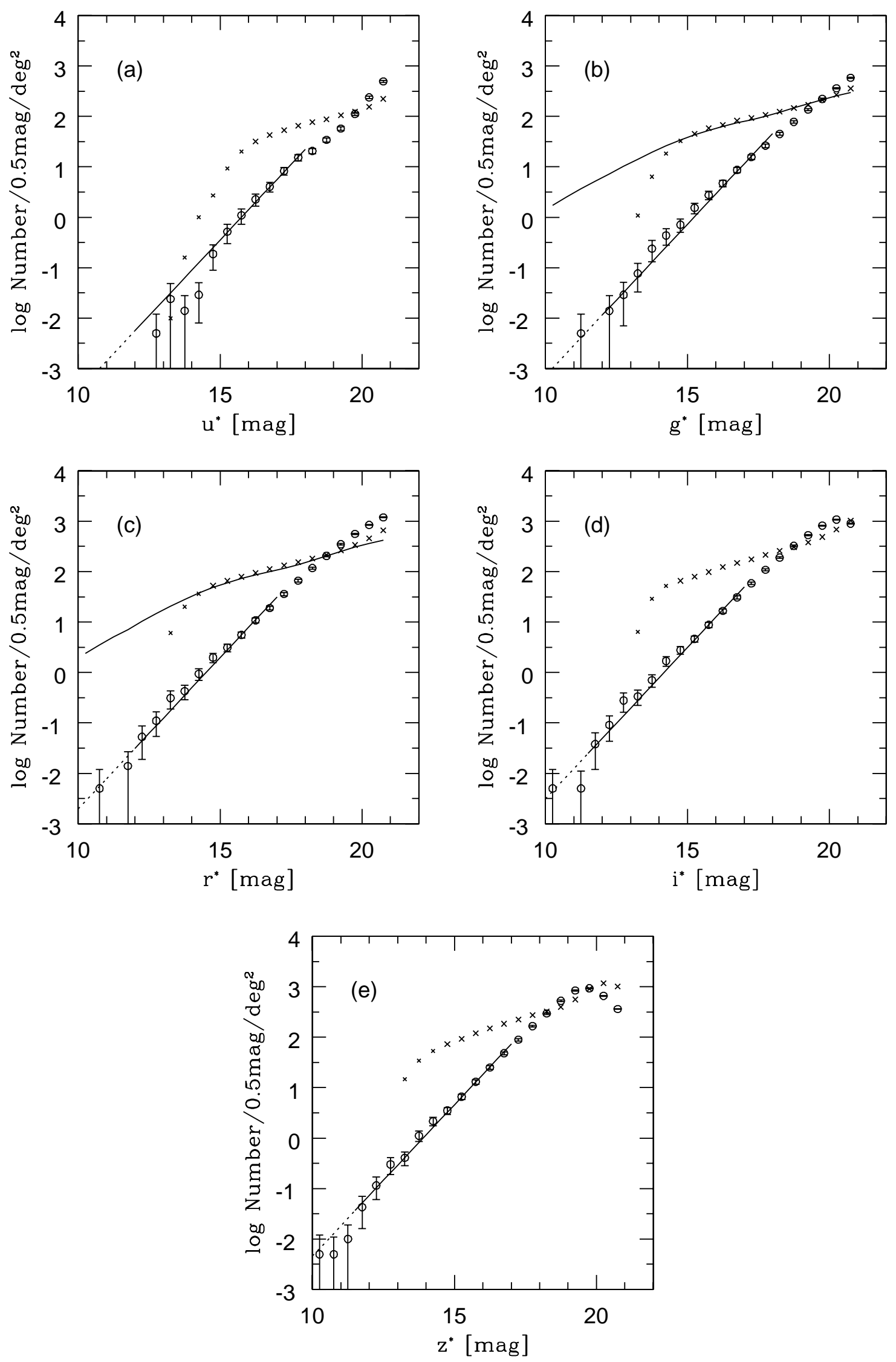

FIG. 13.-Number counts of galaxies as a function of magnitude in the five color bands $u^{\prime}, g^{\prime}, r^{\prime}, i^{\prime}$, and $z^{\prime}$ for the southern equatorial stripe. Open points show the galaxy counts from the machine-selected sample. The error bars include contributions from both shot-noise and large-scale structure (see text for details). The line segment shows the counts-magnitude relation expected in a homogeneous universe with "Euclidean" geometry: $N(m)=A_{\lambda} 10^{0.6 m}$. The crosses show the observed star counts (small crosses: data where stars saturate in the image and therefore suffer from incompleteness), and the solid curve shows the prediction of the Bahcall-Soneira model. 

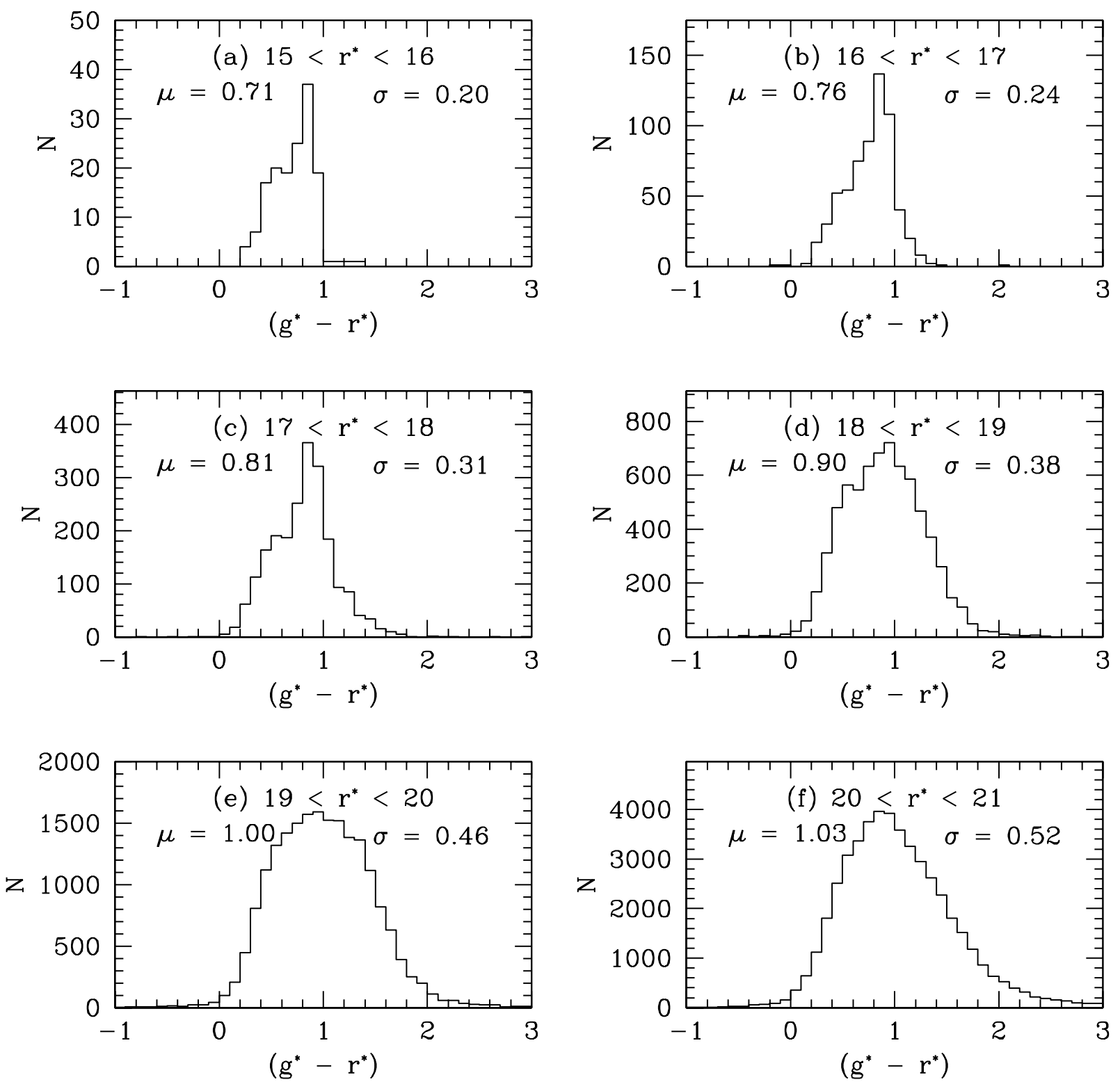

FIG. 14.-Distribution of $g^{*}-r^{*}$ colors of all galaxies in the third CCD column of run 756. The colors are computed from the dereddened Petrosian magnitudes of galaxies. $(a)$ to $(f)$ : This distribution in different bins of $r^{\prime}$ Petrosian magnitude. The mean $(\mu)$ and scatter $(\sigma)$ of the distribution are listed in each panel.

$12<r^{*}<21$. The galaxy number counts are derived from two independent stripes of imaging data along the celestial equator, one each toward the northern and the southern Galactic cap, each covering about $200 \mathrm{deg}^{2}$ of sky. The galaxy counts from both stripes agree very well in the magnitude range $16<r^{*}<21$, implying that we are sampling galaxies in a volume that is large enough to be a fair sample of the universe at these magnitudes. At brighter magnitudes $\left(14<r^{*}<16\right)$, the galaxy counts from the two stripes differ by about $30 \%$. This difference is consistent (at the $2 \sigma$ level) with the fluctuations arising from large-scale structure in the galaxy distribution. Additionally, we have used empirically determined color transformations to translate galaxy counts in the SDSS color bands into those in the $B$ and $I_{814}$ bands. We obtained the $B$-band local luminosity density, $\mathscr{L}_{B}=2.4 \pm 0.4 \times 10^{8} L_{\odot} h \mathrm{Mpc}^{-3}$, for a reasonably wide range of parameters of the Schechter luminosity function in the $B$ band.
At the bright magnitudes, where cosmological and evolutionary corrections are relatively small, the shape of the galaxy number counts-magnitude relation is well characterized by $N\left(m_{\lambda}\right) \propto 10^{0.6 m_{\lambda}}$, the relation expected for a homogeneous galaxy distribution in a universe with "Euclidean" geometry. We have used the amplitude of this relation $\widetilde{A}_{\lambda}$ to determine the luminosity density of the universe at zero redshift in all the five SDSS bands and in the $B$ band (see Table 5).

The results presented in this paper are based on only a fraction of the imaging data taken during the commissioning phase of the SDSS. However, given the high rate of imaging of the SDSS (of about $20 \mathrm{deg}^{2} \mathrm{hr}^{-1}$ ), we already have large sufficient sky coverage from only a few nights of observations to place interesting constraints on such fundamental quantities as the counts-magnitude relation and the luminosity density of the universe. The uniform photometric accuracy and large dynamic range of the CCDs, 

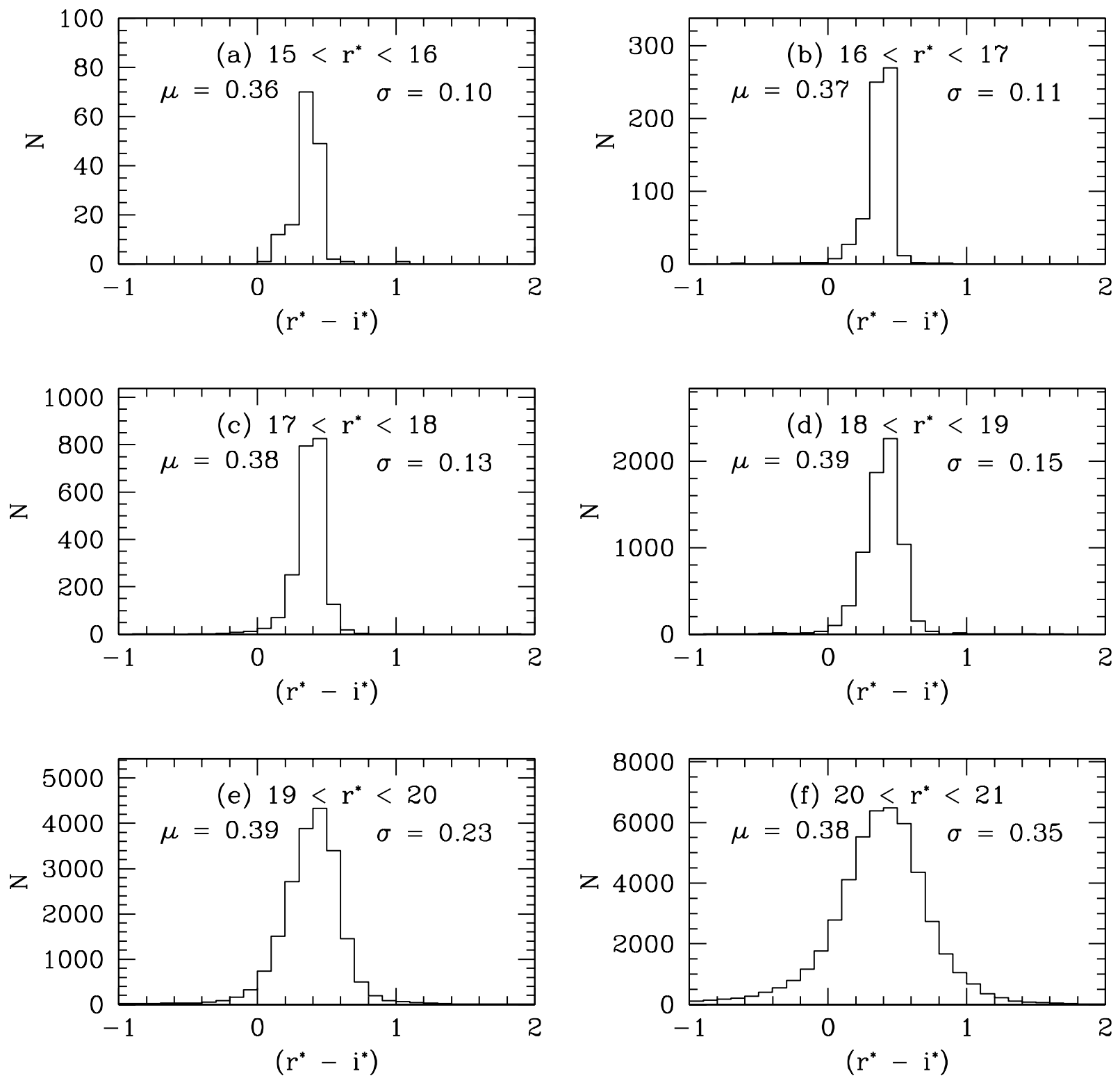

FIG. 15.-Distribution of $r^{*}-i^{*}$ colors of all galaxies in the third CCD column of run 756. The colors are computed from the dereddened Petrosian magnitudes of galaxies. $(a)$ to $(f)$ : This distribution in different bins of $r^{*}$ Petrosian magnitude. The mean $(\mu)$ and scatter $(\sigma)$ of the distribution are listed in each panel.

coupled with the high rate of imaging of the SDSS will soon enable studies of both the properties and the large-scale distribution of galaxies at unprecedented accuracy.

The errors in the galaxy number counts and the luminosity density of the universe in different bands derived in this work are still dominated by fluctuations due to largescale structure. The full SDSS survey will increase the sky coverage by a factor of 25 and, more importantly, will enable the study of field-to-field variation of bright galaxy counts. Since the fractional error in galaxy counts due to large-scale structure is inversely proportional to sky coverage, we can expect to reduce this error (and the error in the luminosity density of the universe) by a similar factor.

M. F. acknowledges support in Tokyo from a grant-inaid from the Ministry of Education of Japan and in Prince- ton from the Raymond and Beverly Sackler Fellowship. M. A. S. acknowledges support from Research Corporation and NSF grants AST 96-18503 and AST 00-71091. I. S. was supported by the NASA grant NAG 5-3364. The Sloan Digital Sky Survey (SDSS) is a joint project of the University of Chicago, Fermilab, the Institute for Advanced Study, the Japan Participation Group, the Johns Hopkins University, the Max Planck Institute for Astronomy, New Mexico State University, Princeton University, the US Naval Observatory, and the University of Washington. Apache Point Observatory, site of the SDSS telescopes, is operated by the Astrophysical Research Consortium (ARC). Funding for the project has been provided by the Alfred P. Sloan Foundation, the SDSS member institutions, the National Aeronautics and Space Administration, the National Science Foundation, the Department of Energy, Monbusho, and the Max Planck Society. 


\section{APPENDIX}

\section{ANGULAR CROSS-CORRELATION BETWEEN GALAXIES IN DIFFERENT MAGNITUDE BINS}

The relation between the angular two-point autocorrelation function $w_{\mathrm{a}}(\theta)$ computed from a flux-limited galaxy photometric catalog and the spatial two-point correlation function $\xi(r)$ is (Limber 1953; Peebles 1993)

$$
w_{a}(f, \theta)=\frac{\iint\left(r_{1} r_{2}\right)^{2} d r_{1} d r_{2} \psi\left(r_{1}\right) \psi\left(r_{2}\right) \xi\left(r_{12}\right)}{\left[\int r^{2} d r \psi(r)\right]^{2}},
$$

where $f$ is the flux limit of the photometric catalog,

$$
r_{12}=\sqrt{r_{1}^{2}+r_{2}^{2}+2 r_{1} r_{2} \cos (\theta)}
$$

is the distance between the points $r_{1}$ and $r_{2}$, and the selection function $\psi(r)$ is related to the galaxy luminosity function $\phi(L)$ by

$$
\psi(r)=\int_{4 \pi r^{2} f}^{\infty} \phi(L) d L .
$$

Note that in deriving equation (A2) we have assumed that the galaxy clustering is independent of luminosity. The angular autocorrelation function $w_{a}\left(f_{1}, f_{2}, \theta\right)$ of galaxies with fluxes in the range $f_{2}<f<f_{1}$ is similar to equation (A1), but with the selection function defined by

$$
\psi(r)=\int_{4 \pi r^{2} f_{2}}^{4 \pi r^{2} f_{1}} \phi(L) d L .
$$

The two-point angular cross-correlation between galaxies in two different magnitude bins corresponding to flux ranges $f_{2}<f<f_{1}$ and $f_{4}<f<f_{3}$ is

$$
w_{c}\left(f_{1}, f_{2}, f_{3}, f_{4}, \theta\right)=\frac{\iint\left(r_{1} r_{2}\right)^{2} d r_{1} d r_{2} \psi_{1}\left(r_{1}\right) \psi_{2}\left(r_{2}\right) \xi\left(r_{12}\right)}{\int r^{2} d r \psi_{1} \int r^{2} d r \psi_{2}}
$$

The two selection functions are

$$
\psi_{1}(r)=\int_{4 \pi r^{2} f_{2}}^{4 \pi r^{2} f_{1}} \phi(L) d L, \psi_{2}(r)=\int_{4 \pi r^{2} f_{4}}^{4 \pi r^{2} f_{3}} \phi(L) d L .
$$

For equally spaced magnitude bins, $\left(f_{4} / f_{2}\right)=\left(f_{3} / f_{1}\right)$,

$$
\psi_{2}(r)=\psi_{1}\left(r \sqrt{f_{4} / f_{2}}\right) .
$$

For a power-law correlation function of the form $\xi\left(r_{12}\right)=\left(r_{0} / r_{12}\right)^{\gamma}$, equation (A4) becomes

$$
w_{c}\left(f_{1}, f_{2}, f_{3}, f_{4}, \theta\right)=\frac{r_{0}^{\gamma} \iint\left(r_{1} r_{2}\right)^{2} d r_{1} d r_{2} \psi_{1}\left(r_{1}\right) \psi_{1}\left(r_{2} \sqrt{f_{4} / f_{2}}\right) r_{12}^{-\gamma}}{\int r^{2} d r \psi_{1}(r) \int r^{2} d r \psi_{1}\left(r \sqrt{f_{4} / f_{2}}\right)} .
$$

Under the small-angle approximation $(|\theta| \ll 1)$,

$$
r_{12}=\sqrt{\left(r_{2}-r_{1}\right)^{2}+r_{1} r_{2} \theta^{2}}
$$

The numerator in equation (A7) is dominated by small values of $r_{12}$. So, defining

$$
v=\frac{r_{2}-r_{1}}{r_{0}}
$$

and setting $r_{1}=r_{2} \equiv r$ in the numerator of equation (A7), we obtain

$$
w_{c}\left(f_{1}, f_{2}, f_{3}, f_{4}, \theta\right) \approx r_{0}^{v}\left(\frac{f_{4}}{f_{2}}\right)^{3 / 2}\left[\int r^{2} d r \psi_{1}(r)\right]^{-2} \int r^{4} \psi_{1}(r) \psi_{1}\left(r \sqrt{f_{4} / f_{2}}\right) d r \int \frac{d v}{\left[v^{2}+r^{2} \theta^{2}\right]^{\gamma / 2}},
$$

where the integral over $v$ ranges from $-\infty$ to $+\infty$ (since both $r_{1}$ and $r_{2}$ vary between 0 to $+\infty$ ). Now,

$$
\int \frac{d x}{\left(a^{2}+x^{2}\right)^{\gamma / 2}}=a^{1-\gamma} \int \frac{d x}{\left(1+x^{2}\right)^{\gamma / 2}} .
$$

Therefore, equation (A10) becomes

$$
w_{c}\left(f_{1}, f_{2}, f_{3}, f_{4}, \theta\right)=r_{0}^{\gamma} H_{\gamma} \theta^{1-\gamma}\left(\frac{f_{4}}{f_{2}}\right)^{3 / 2} \frac{\int r^{5-\gamma} d r \psi_{1}(r) \psi_{1}\left(r f_{4} / f_{2}\right)}{\left[\int r^{2} d r \psi_{1}(r)\right]^{2}},
$$


where

$$
H_{\gamma}=\int_{-\infty}^{+\infty} \frac{d x}{\left(1+x^{2}\right)^{\gamma / 2}}=\frac{(-1 / 2) !(\gamma / 2-3 / 2) !}{(\gamma / 2-1) !} .
$$

Since $w_{a}\left(f_{1}, f_{2}, \theta\right)=w_{\mathrm{c}}\left(f_{1}, f_{2}, f_{1}, f_{2}, \theta\right)$, equation (A12) can be written as

$$
w_{c}\left(f_{1}, f_{2}, f_{3}, f_{4}, \theta\right)=w_{a}\left(f_{1}, f_{2}, \theta\right)\left(\frac{f_{4}}{f_{2}}\right)^{3 / 2} \frac{\int r^{5-\gamma} \psi_{1}(r) \psi_{1}\left(r \sqrt{f_{4} / f_{2}}\right) d r}{\int r^{5-\gamma}\left[\psi_{1}(r)\right]^{2} d r} .
$$

We use this relation between $w_{c}\left(f_{1}, f_{2}, f_{3}, f_{4}, \theta\right)$ and $w_{a}\left(f_{1}, f_{2}, \theta\right)$ to construct the covariance matrix of the galaxy counts in different magnitude bins. We evaluate the integrals in equation (A14) using the luminosity function in the appropriate SDSS passband derived by Blanton et al. (2000).

Bahcall, J. N., \& Soneira, R. M. 1980, ApJS, 44, 73

Bertin, E., \& Dennefeld, M. 1997, A\&A, 317, 43

Blanton, M., et al. 2001, AJ, 121, 2358

Chen, B., et al. 2001, ApJ, 553, 184

Efstathiou, G., Ellis, R. S., \& Peterson, B. A. 1988, MNRAS, 232, 431

Finlator, K., et al. 2000, AJ, 120, 2615

Folkes, S., et al. 1999, MNRAS, 308, 459

Fukugita, M., Hogan, C. J., \& Peebles, P. J. E. 1998, ApJ, 503, 518

Fukugita, M., Ichikawa, T., Gunn, J. E., Doi, M., Shimasaku, K., \& Schneider, D. P. 1996, AJ, 111, 1748

Fukugita, M., Ichikawa, T., \& Sekiguchi, M. 2001, in preparation

Fukugita, M., Shimasaku, K., \& Ichikawa, T. 1995, PASP, 107, 945

Gardner, J. P., Sharples, R. M., Carrasco, B. E., \& Frenk, C. S. 1996, MNRAS, 282, L1

Gunn, J. E., et al. 1998, AJ, 116, 3040

Hall, P., \& Mackay, C. D. 1984, MNRAS, 210, 979

Kennicutt, R. C. 1992, ApJS, 79, 255

Koo, D. C., \& Kron, R. G. 1992, ARA\&A, 30, 613

Landolt, A. U. 1992a, AJ, 104, 340 1992b, AJ, 104, 372

Lilly, S. J., Cowie, L. L., \& Gardner, J. P. 1991, ApJ, 369, 79

Limber, D. N. 1953, ApJ, 117, 134

Loveday, J., Peterson, B. A., Efstathiou, G., \& Maddox, S. J. 1992, ApJ, 390,338

\section{REFERENCES}

Lupton, R H., et al. 2001, in preparation

Maddox, S. J., Sutherland, W. J., Efstathiou, G., Loveday, J., \& Peterson, B. A. 1990, MNRAS, 247, P1

Metcalfe, N., Shanks, T., Fong, R., \& Jones, L. R. 1991, MNRAS, 249, 498

Peebles, P. J. E. 1993, Principles of Physical Cosmology (Princeton: Princeton Univ. Press)

Petrosian, V. 1976, ApJ, 209, L1

Postman, M., Lauer, T. R., Szapudi, I., \& Oegerle, W. 1998, ApJ, 506, 33

Ratcliffe, A., Shanks, T., Parker, Q. A., \& Fong, R. 1998, MNRAS, 293, 197

Ratnatunga, K. U., Griffiths, R. E., \& Ostrander, E. J. 1999, AJ, 118, 86

Sandage, A. 1988, ARA\&A, 26, 561

Santiago, B. X., Strauss, M. A., Lahav, O., Davis, M., Dressler, A., \& Huchra, J. P. 1996, ApJ, 461, 38

Schechter, P. L. 1976, ApJ, 203, 297

Schlegel, D. J., Finkbeiner, D. P., \& Davis, M. 1998, ApJ, 500, 525

Scranton, R., et al. 2000, BAAS, 196, 5505

Shimasaku, K., et al. 2001, AJ, 122, 1238

Smith, J. A., et al. 2000, BAAS, 197, 1311

Tyson, J. A. 1988, AJ, 96, 1

Weir, N., Djorgovski, S., \& Fayyad, U. M. 1995, AJ, 110, 1

York, D. G., et al. 2000, AJ, 120, 1579

Zucca, E., et al. 1997, A\&A, 326, 477 